\title{
The Effect of Pilates Training and Electrical Stimulation on Motor and Cognitive Function of Women with Multiple Sclerosis
}

\author{
Bahloul Ghorbanian ${ }^{1 *}$, Yousef Saberi², Maryam Rasouli ${ }^{3}$ \\ ${ }^{1}$ Department of Sport Sciences, Faculty of Education and Psychology, Azarbaijan Shahid Madani University, Tabriz. Iran \\ ${ }^{2}$ Department of Exercise Physiology and Corrective Exercises, Faculty of Sport Sciences, Urmia University, Urmia, Iran \\ ${ }^{3}$ Department of Sport Sciences, Azarbaijan Shahid Madani University, Tabriz. Iran
}

\section{ABSTRACT}

Introduction: Multiple Sclerosis (MS) is a chronic, debilitating nervous system disease, which damages the myelin of the central nervous system. The purpose of this study was to investigate the effect of 8 weeks Pilates training and direct transcranial stimulation (tDCS) on motor and cognitive functions in women with MS. Materials and Methods: The present study was a semi-experimental one in which the women with MS in Tabriz, Iran were selected and 36 of them were selected as subjects with age range of 20-40 years. They were randomly divided into four groups; Pilates + tDCS, Pilates + Placebo, tDCS, and control groups. The Pilates and tDCS training program consisted of 8 weeks (three sessions/60 minutes) Pilates training plus 20 minutes of electrical stimulation. Results: The results have shown that after exercise and electrical stimulation, the tDCS + exercise and exercise + placebo groups improved motor functions (general endurance, general speed, flexibility, balance). The results also revealed that among the measured cognitive factors, there was a significant improvement in the cognitive flexibility in the tDCS + exercise, the exercise + placebo, and the tDCS groups. Conclusion: Considering the combined effects of exercise with tDCS on motor functions, it seems that the use of these two techniques is helpful in improving motor functions in MS patients. However, this combined therapy can not improve cognitive functions of patients with MS.

\section{Key words:}

1. Multiple Sclerosis

2. Transcranial Direct

Current Stimulation

3. Cognition

*Corresponding Author: Bahloul Ghorbanian

E-mail:b.ghorbanian@azaruniv.ac.ir 


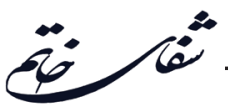

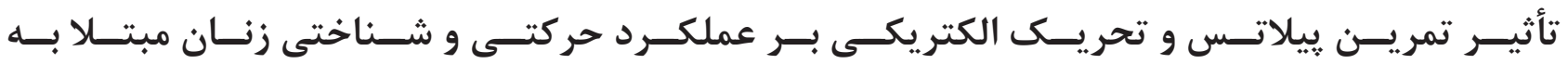

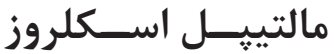

\author{
بهلول قربانيان"، يوسف صابرى ‘، مريم رسولى" \\ 'اَروه علوم ورزشى، دانشكده علوم تربيتى و روانشناسى، دانشعاه شهيد مدنى آذربايجان، تبريز، ايران

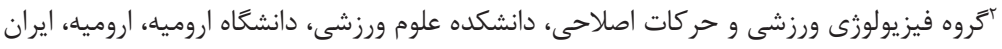 \\ "كروه علوم ورزشى، دانشكاه شهيد مدنى آذربايجان، تبريز، ايران
}

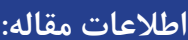

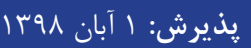

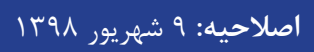

دريافت: זץ ارديبهشت ג

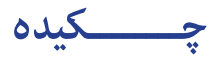

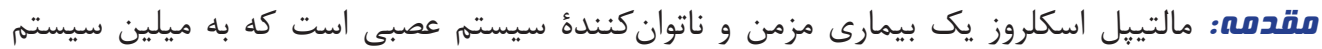

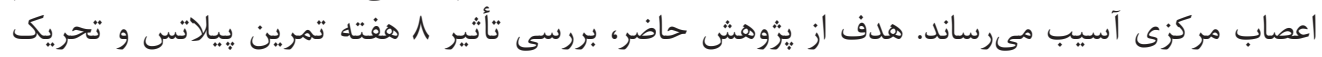

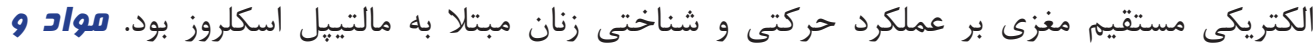

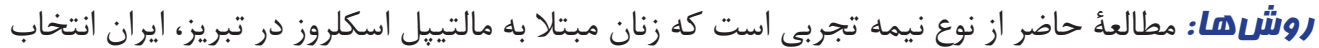

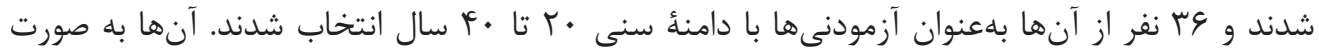

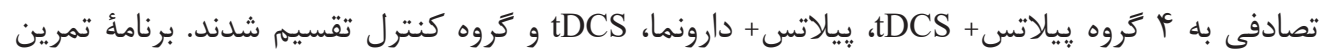

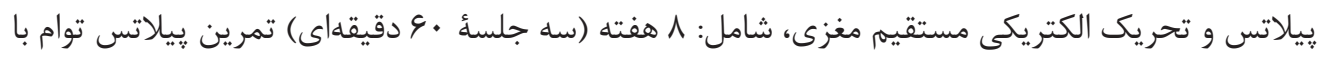

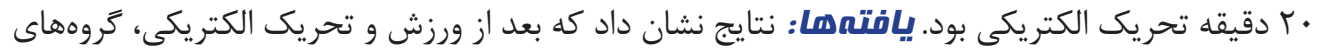

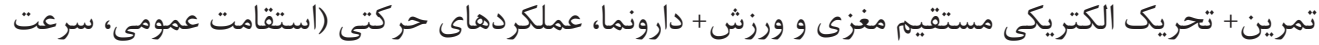

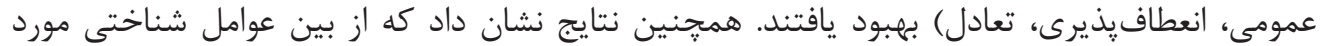

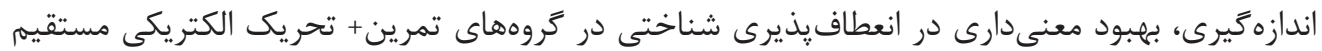

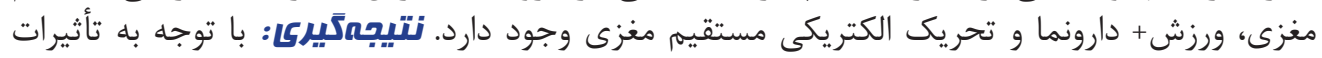

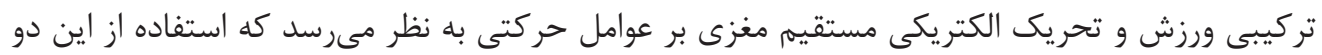

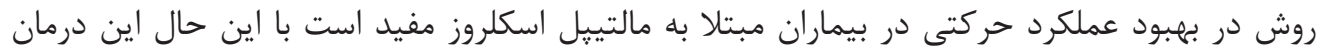

كليد وازهها:

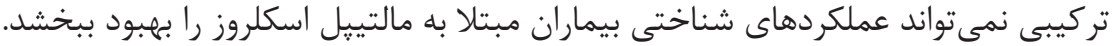

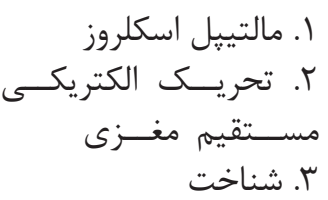

" مويسنده مسئول: بهلول قربانيان

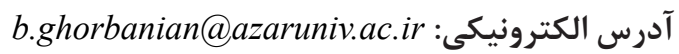




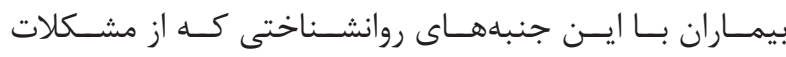

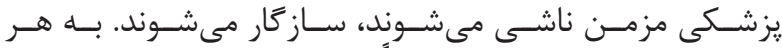

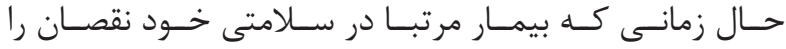

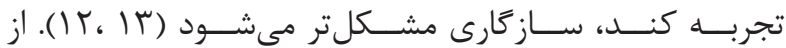

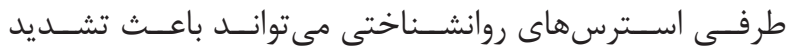

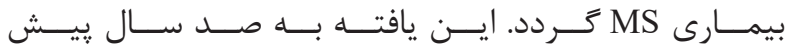

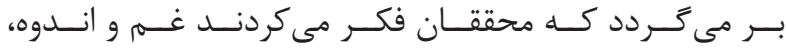

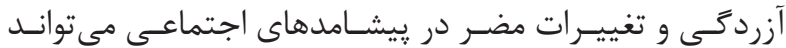

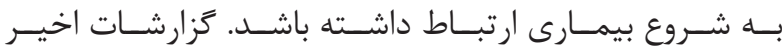

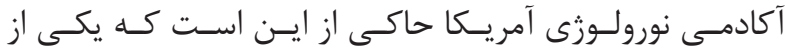

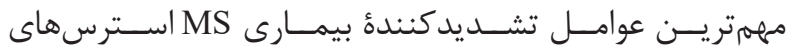

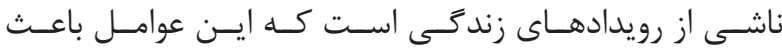

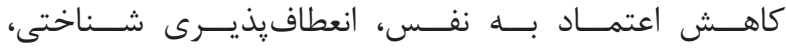

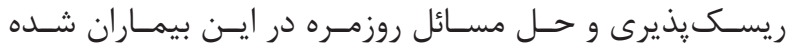

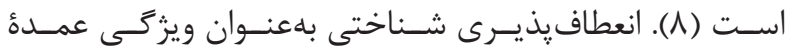

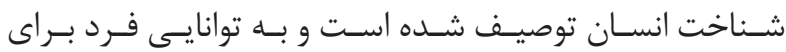

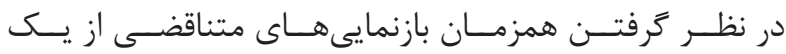

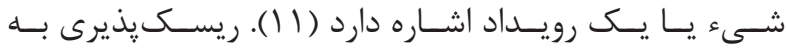

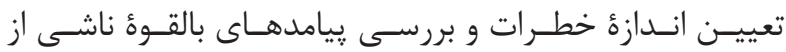

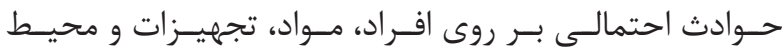

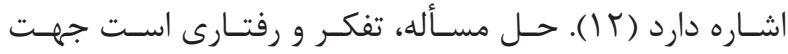

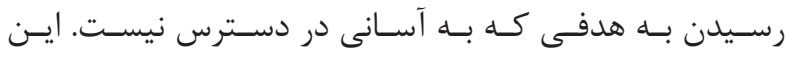

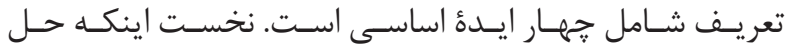

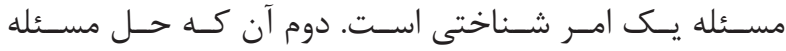

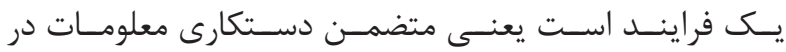

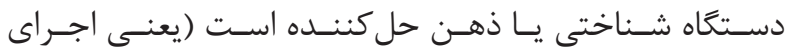

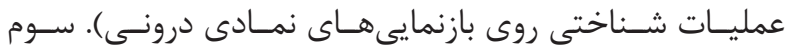

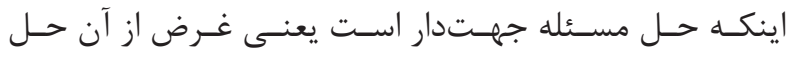

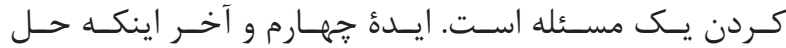

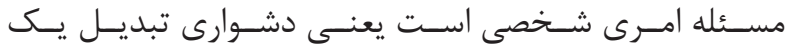

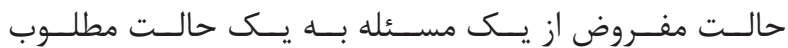

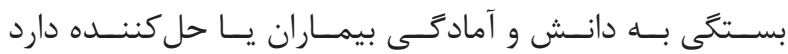

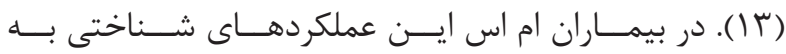

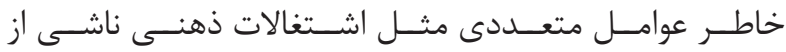

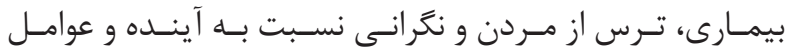

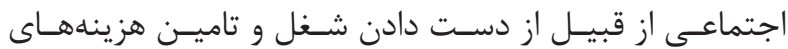

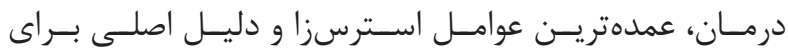

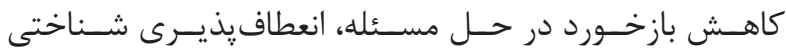

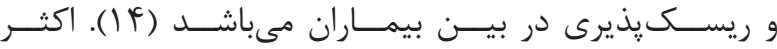

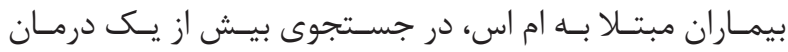

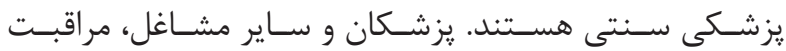

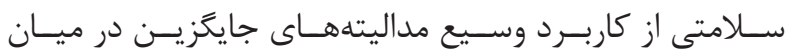

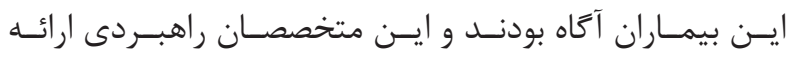

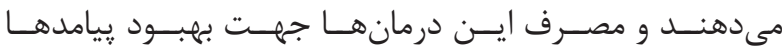
راكنتــرل مىنماينــد.

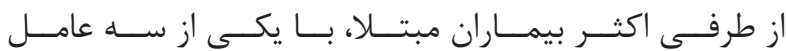

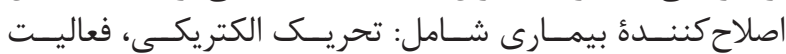

مقدمه

مالتييـل اسـكلروز (MS)' يـك بيمـارى مزمــن خــود ايمنسى

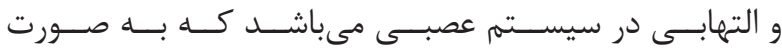

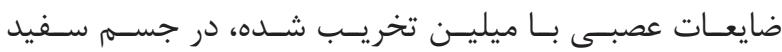

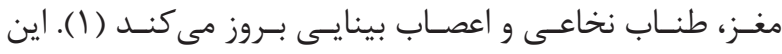

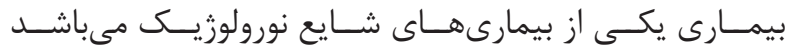

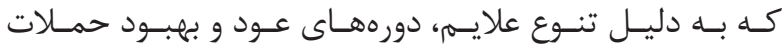

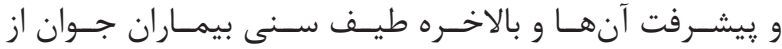

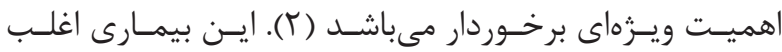

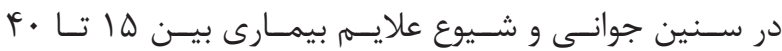

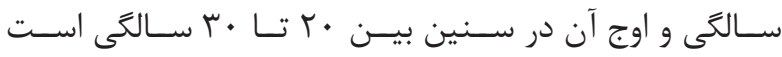

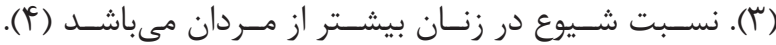

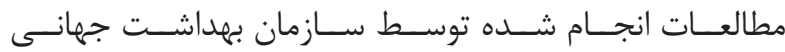

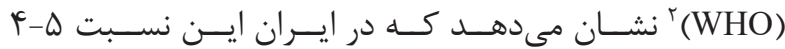

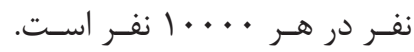

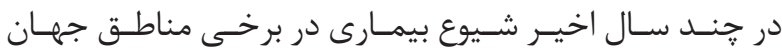

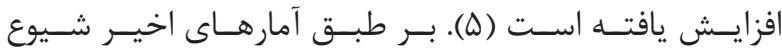

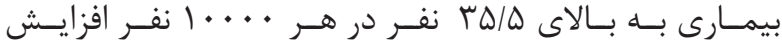

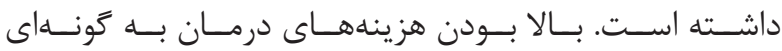

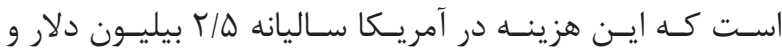

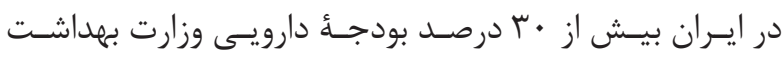

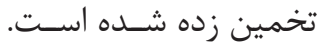

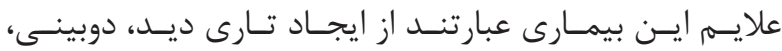

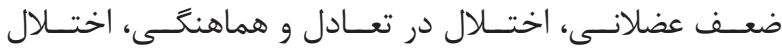

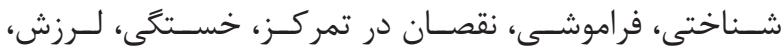

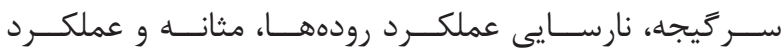

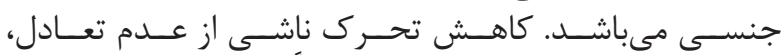

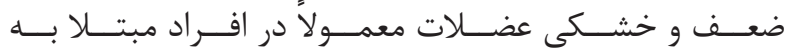

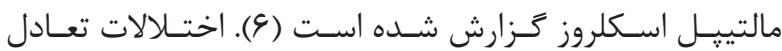

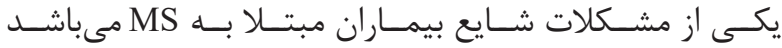

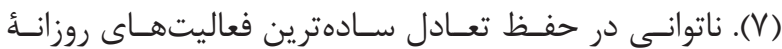

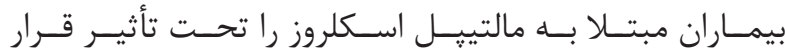

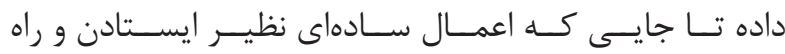

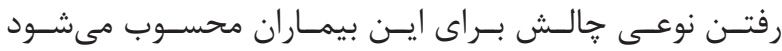

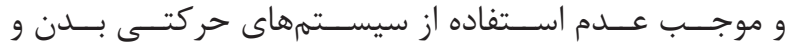

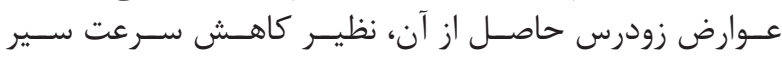

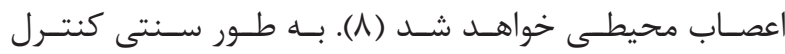

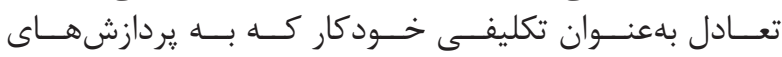

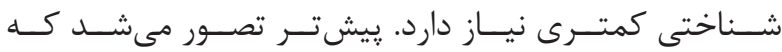

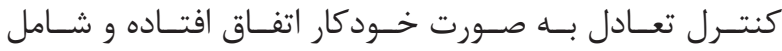

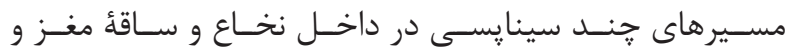

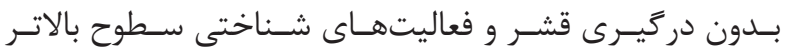

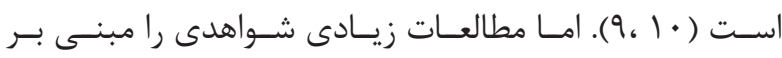

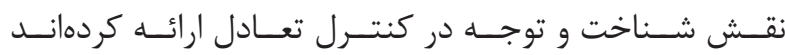

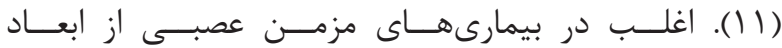

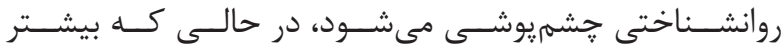




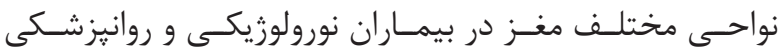

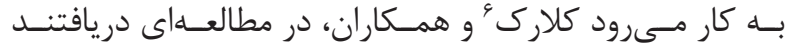

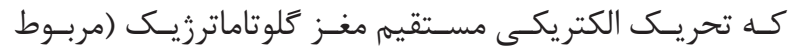

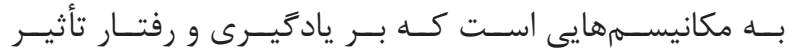

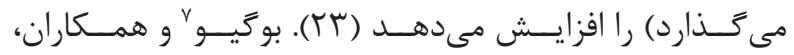

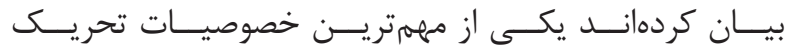

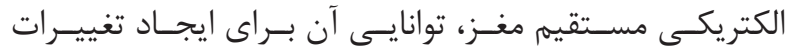

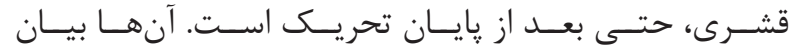

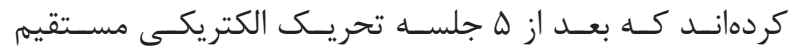

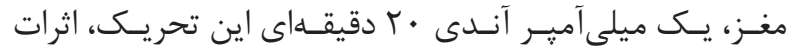

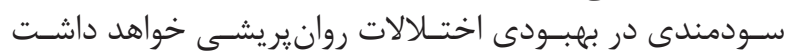

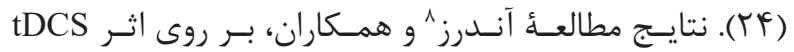

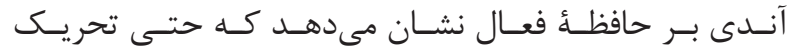

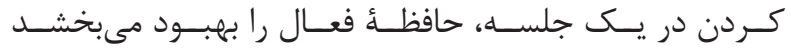

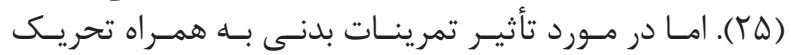

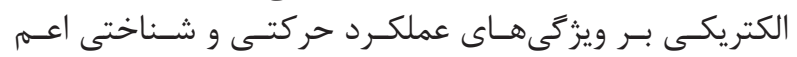

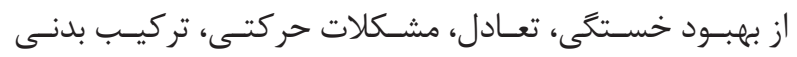

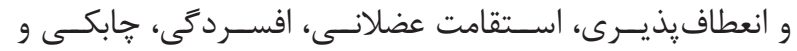

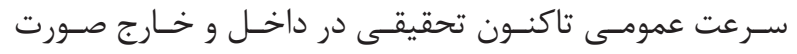

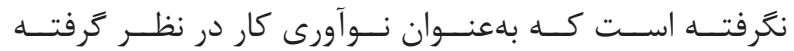

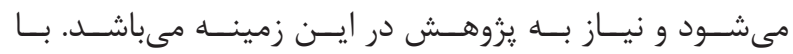

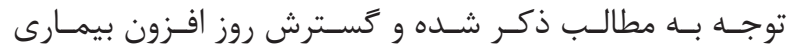
MS

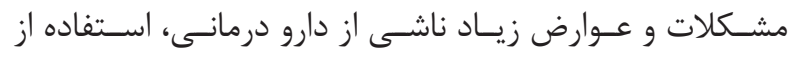

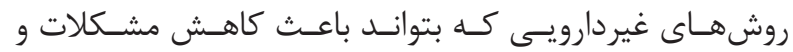

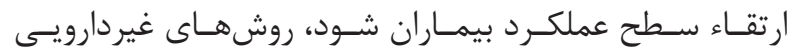

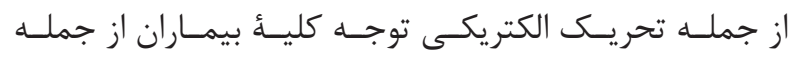

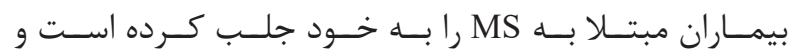

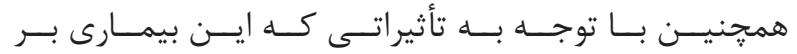

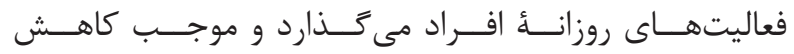

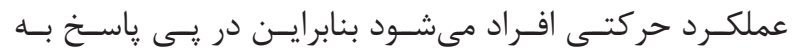

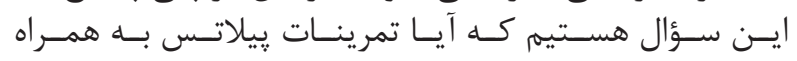

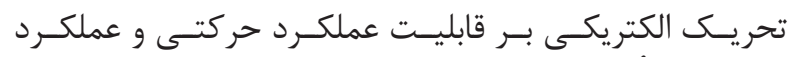

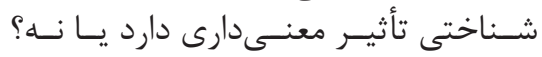

مواد و روشها - - ماد

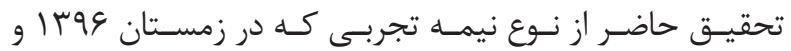

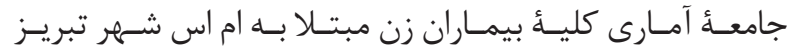

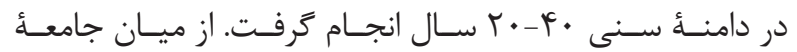

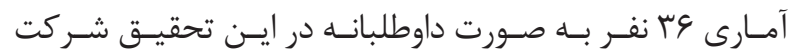

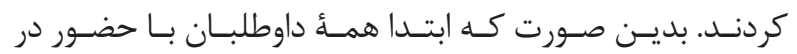

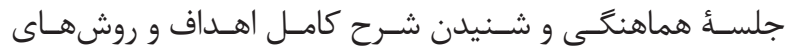

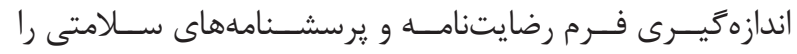

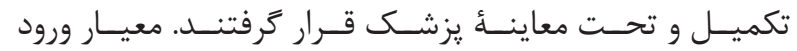

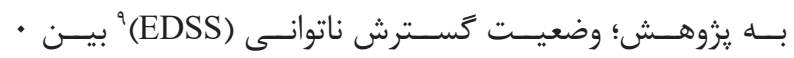

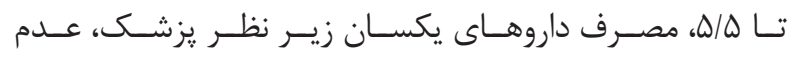

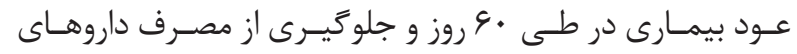

${ }^{3}$ Romberg

${ }^{4}$ Rampello

${ }^{5}$ Transcranial direct-current stimulation

${ }^{6}$ Klarc

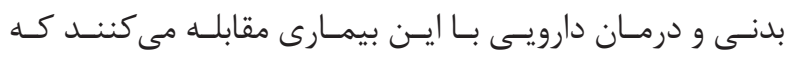

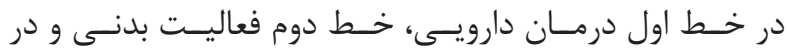

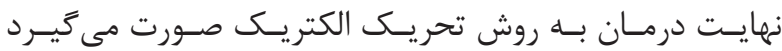

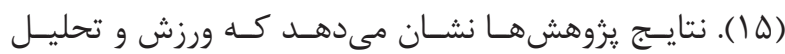

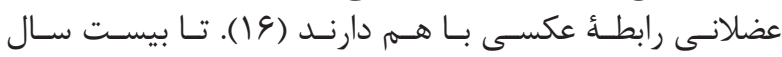

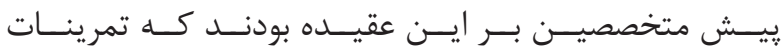

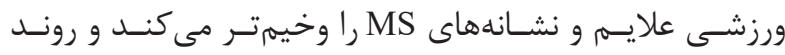

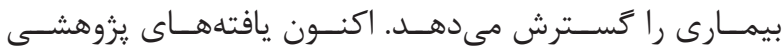

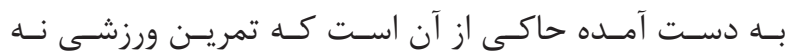

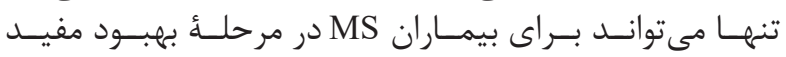

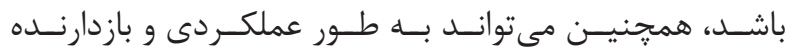

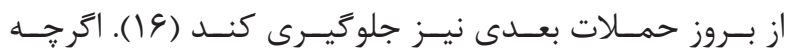

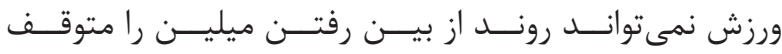

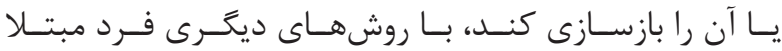

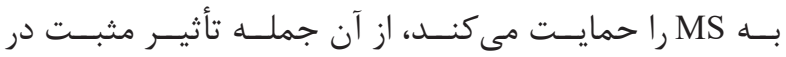

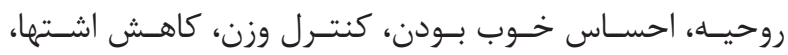

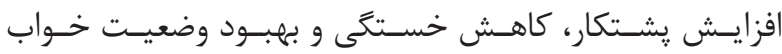

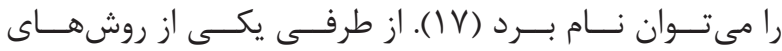

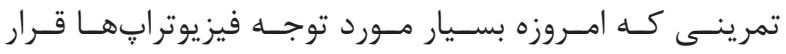

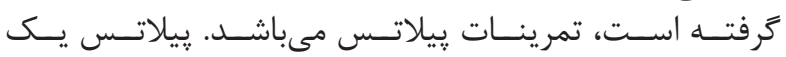

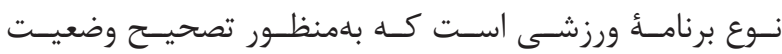

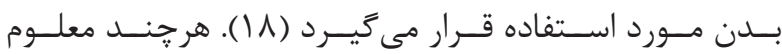

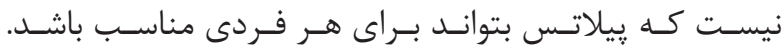

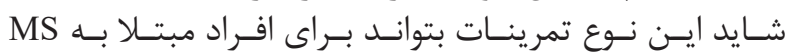

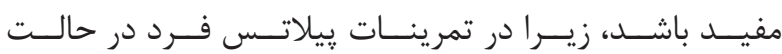

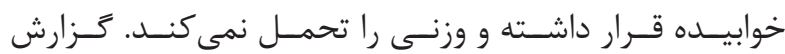

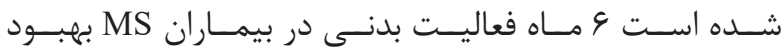

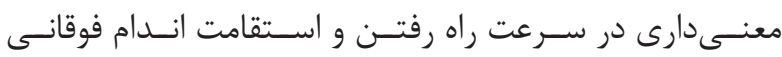

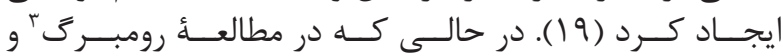

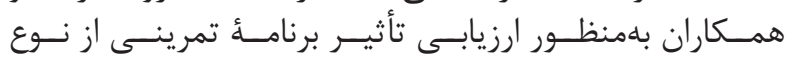

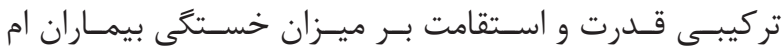

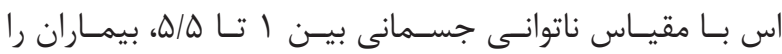

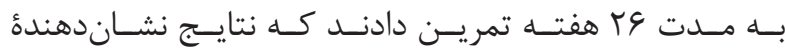

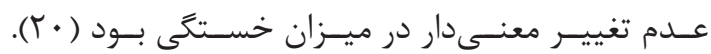

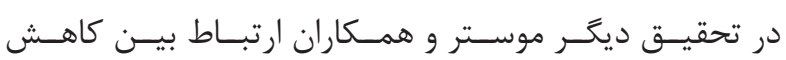

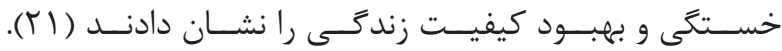

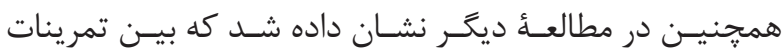

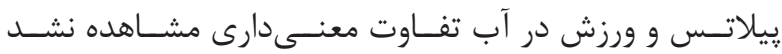

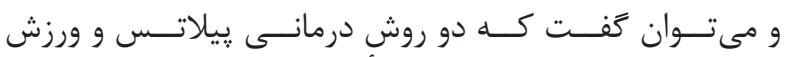

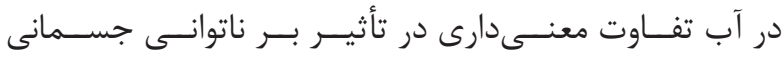

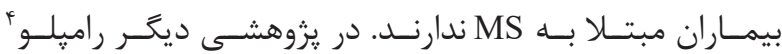

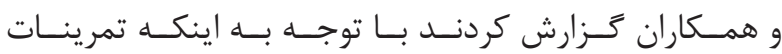

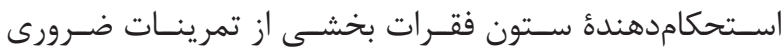

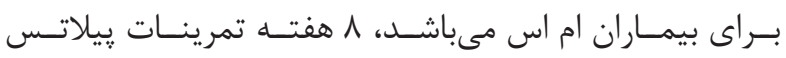

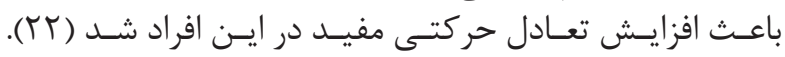

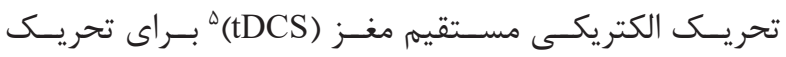

\section{${ }^{7}$ Bogio}

${ }^{8}$ Anders

${ }^{9}$ Expanded disability status scale 


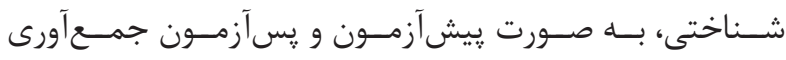

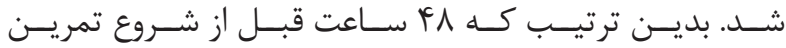

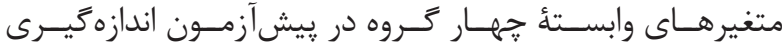

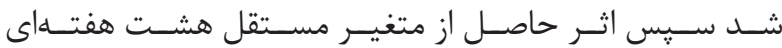

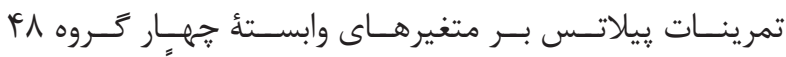

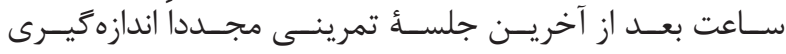

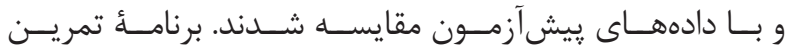

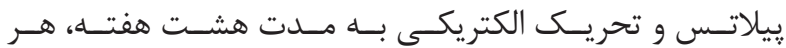

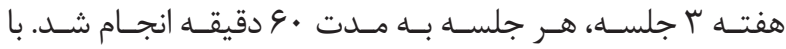

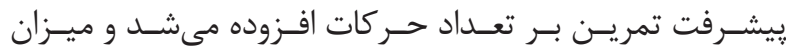

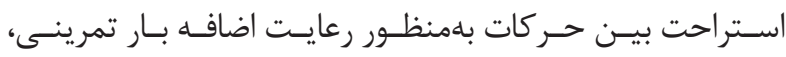

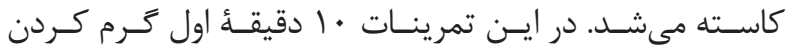

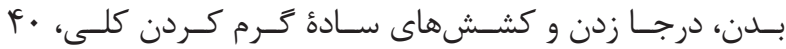

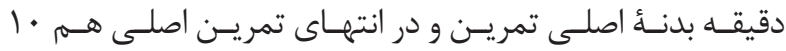

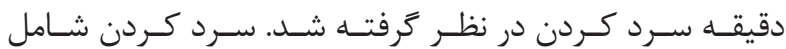

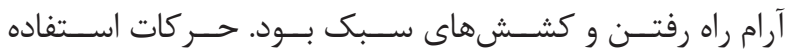

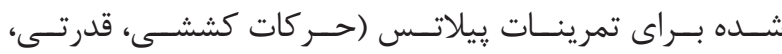

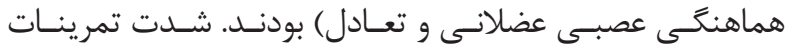

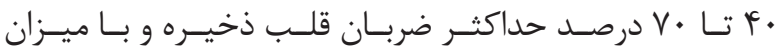

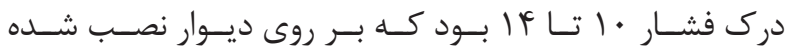

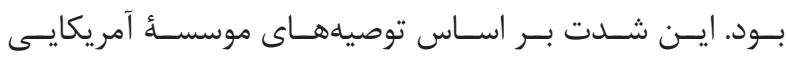

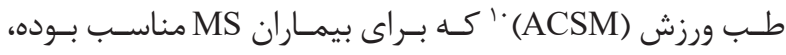

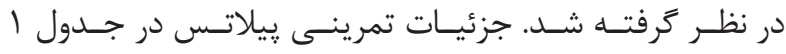

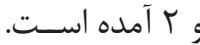

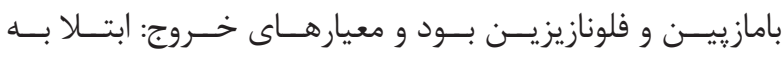

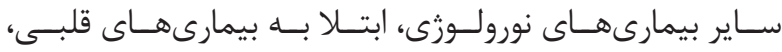

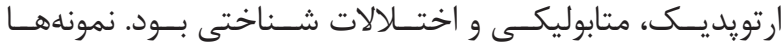

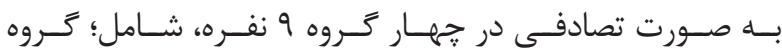

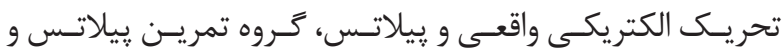

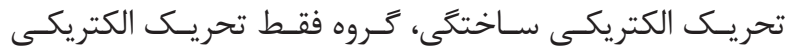

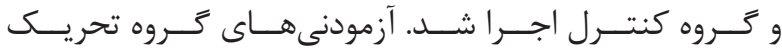

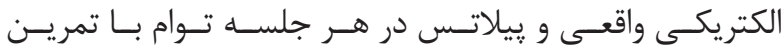

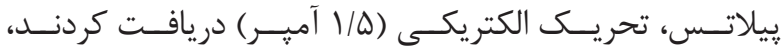

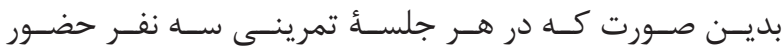

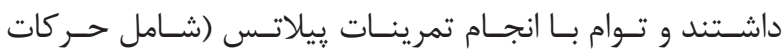

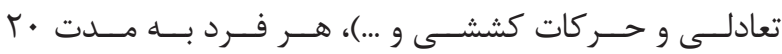

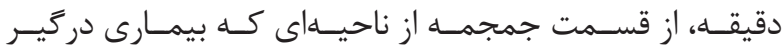

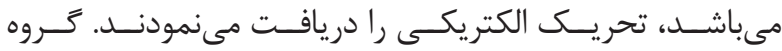

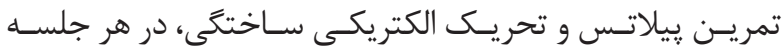

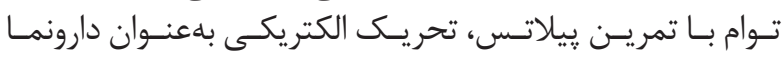

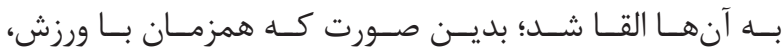

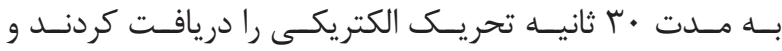

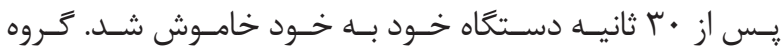

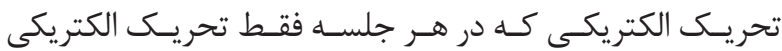

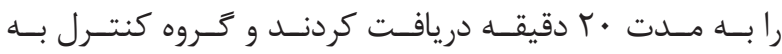

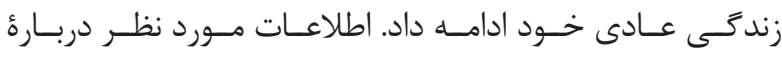

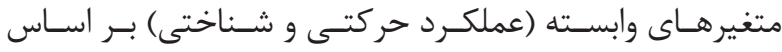

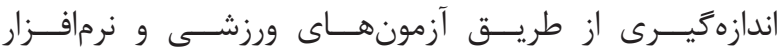

\begin{tabular}{|c|c|}
\hline \multicolumn{2}{|r|}{ تمرينات } \\
\hline صندلى تايى یا باز (Openleg reckre) & هرخش يك يا (Single leg circles) \\
\hline كشش عنكبوتى (Spin stretch) & غلتيدن بالا تنه به جلو (Roll up) \\
\hline قو (Saw) & صدتايى (The hundered) \\
\hline ضربه به يهلو (Side- kicks-serie) & كشش يك یا (Single leg stretch) \\
\hline ضربئ دو یا (Double leg nick) & كشش دو يا (Double leg stretch) \\
\hline رقص عنكبوت (Spin) & ضربدرى (Criss-Cross) \\
\hline شنا (Swimming) & كشش دويا صاف (Double strainght leg stretch) \\
\hline ضربه به يهلو روى زانو (Kneeling side kick) & كشش يك يا صاف (قيجى)(Single strainght leg stretch) \\
\hline
\end{tabular}

جدول r- بروتكل تمرينى بِيلاتس (* استراحت بين ستها).

\begin{tabular}{|c|c|c|c|c|c|c|c|c|c|}
\hline سرد كردن & هفتئ & Vis & هiته 9 & هis هند ه & هفتئ P P & هنتة ب & 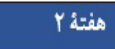 & هفته & كرمثردن \\
\hline هر جلسه & م" مقيقه & . p دقيقه & . p دقيقه & . p. دقيقه & . p. دقيقه & 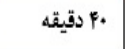 & 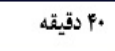 & rp دقيقه & هر جلسه +1 \\
\hline . اقيقه & واحركت & |واحركت & واحركت & هاحركت & هاهركت & |F |حركت & أحركت & |زاحركت & تقيقه \\
\hline مركات & זاتكرار & التكرار & التكرار & . اتكرار. & و تكرار & 1 ثكرار & ل V تكرار & V V Vترار & مركات \\
\hline كششى & با دقيقه & rا دقيقه & rا دقيقه & r/ ب دقيقه & r/ ب دقيقه & r/r دقيقه & F أ دقيقه & |مقيقه ه" & كششىو \\
\hline آرامر راه & لا ست & لا ست & r است & لا ست & لبا ست & لا ست & با ست & لا ست & رجازدن \\
\hline رغتين & \$ \$ درصد -V. & 90-49 درصد & ه 4 د درصد & . & 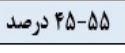 & 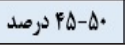 & 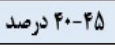 & PQ +F" درصد & \\
\hline
\end{tabular}

${ }^{10}$ The American college of sports medicine 


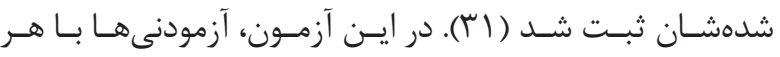

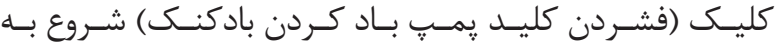

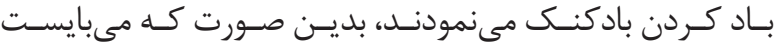

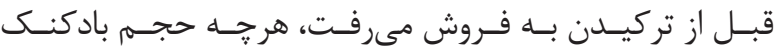

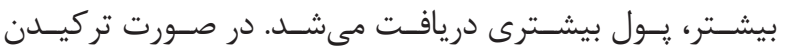

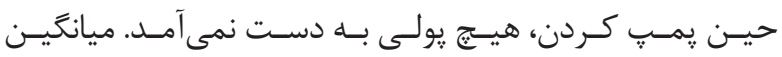

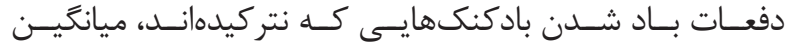

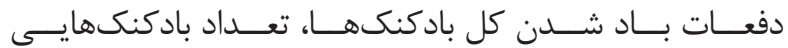

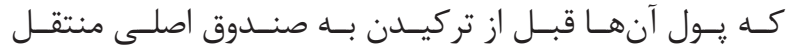

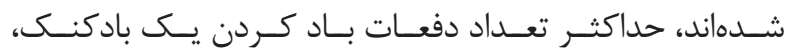

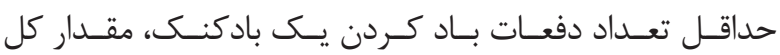

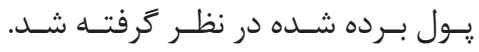

انعطاف بذيرى شناختى

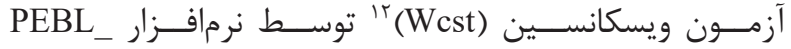
Setup 14

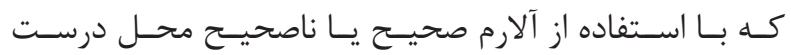

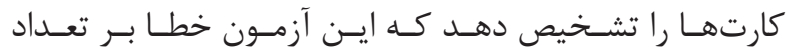

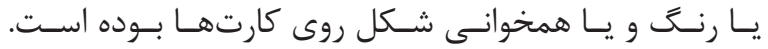

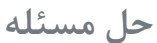

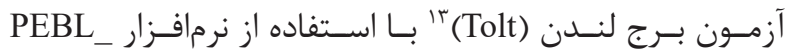

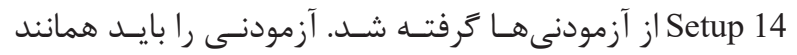

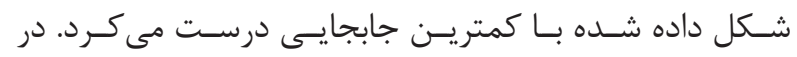

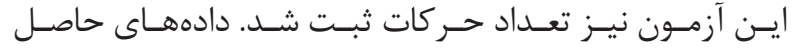

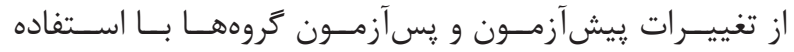

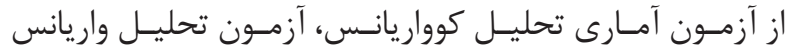

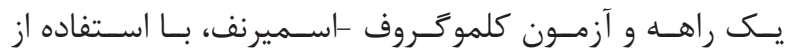

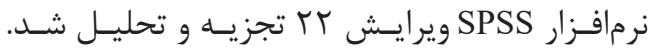

بافته ها

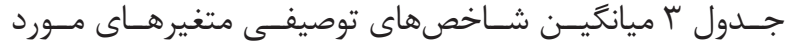

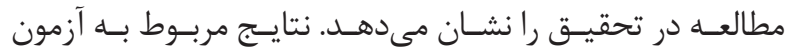

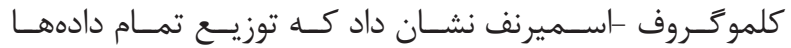

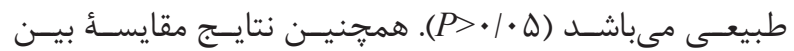

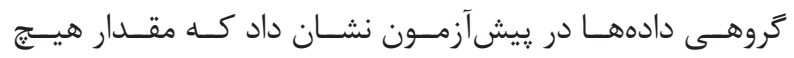

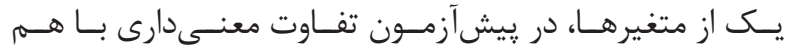

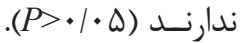

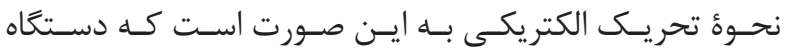

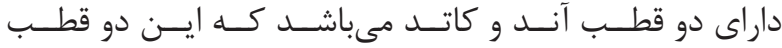

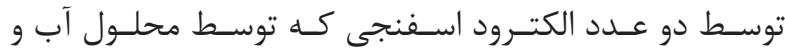

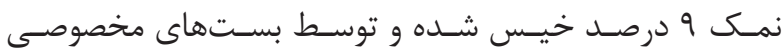

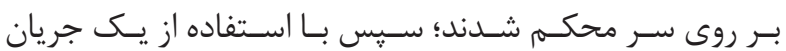

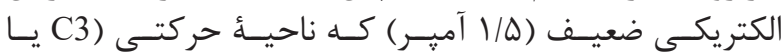

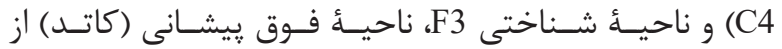

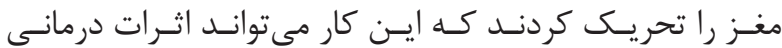

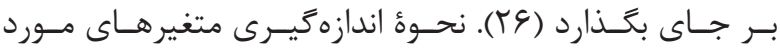

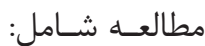

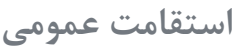

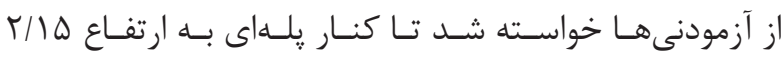

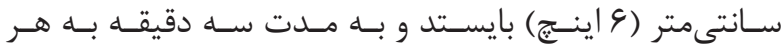

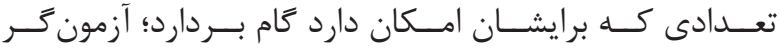

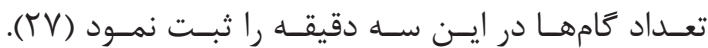
سرعت عمومى - (آ س

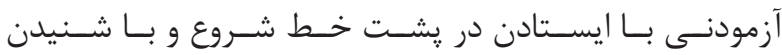

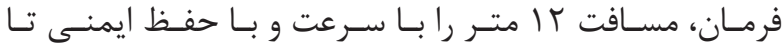

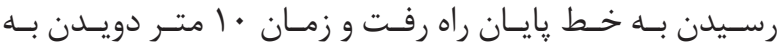

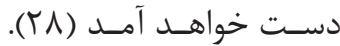
انعطاف يذيرى حركتى

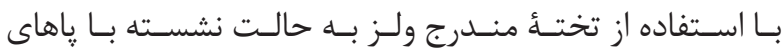

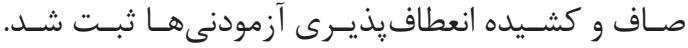

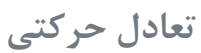

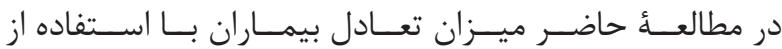

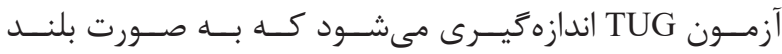

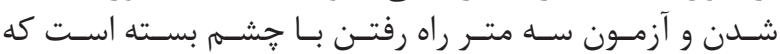

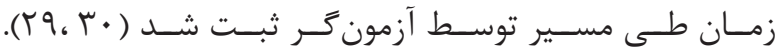

$$
\text { ريسك يذيرى }
$$

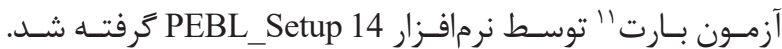

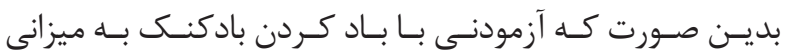

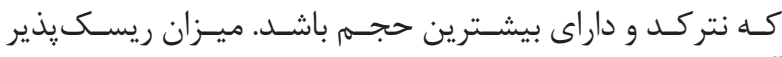

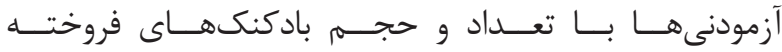

\begin{tabular}{|c|c|c|c|c|}
\hline $\begin{array}{c}\text { كروه كنترل } \\
\text { (9 نفر) }\end{array}$ & $\begin{array}{l}\text { tDCS كروه (9 نفر) } \\
\text { (9 }\end{array}$ & 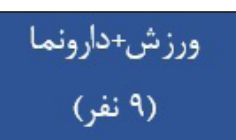 & 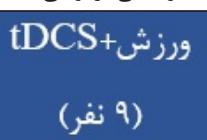 & كروه \\
\hline$q \pm T \Delta / T F$ & $\Gamma \leftarrow / \wedge q \pm \Delta / / 1)$ & $r \varepsilon \pm r$ & $r \Delta / r r \pm F / \Delta \Lambda$ & سن (سال) \\
\hline $\mid \Delta \Lambda \pm N / \Lambda$. & $\mid \Delta Q / \Delta \varphi \pm q / q 1$ & $19 \cdot / 19 \pm 1 \cdot / 4 V$ & $|\varepsilon| / r r \pm \varepsilon \mid \varepsilon \Delta$ & قد (سانتىمتر) \\
\hline$G F / \Delta) \pm I Q / \Lambda T$ & 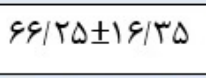 & $99 / V T \pm 1 \cdot / r q$ & $G N / T F \pm V / D$ & وزن (كيلوكرم) \\
\hline$F / T r \pm \cdot / \Lambda Q$ & $\Gamma / \Delta \Delta \pm 1 / / \Gamma$ & $T / T r \pm 1 / T r$ & $\Gamma / 99 \pm 1 / 41$ & EDSS \\
\hline
\end{tabular}

جدول ץ- ميانكَين متغيرهاى توصيفى آزمودنىهاى مورد مطالعه.

\footnotetext{
${ }^{11}$ Baret

${ }^{12}$ Wisconsin card sorting test

${ }^{13}$ Tower of London test
} 


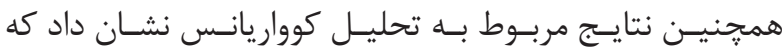

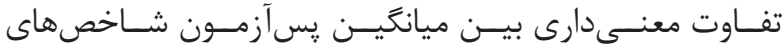

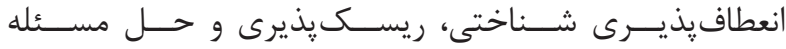

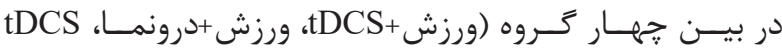

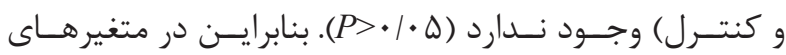

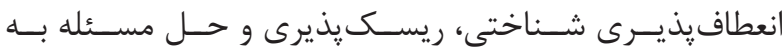

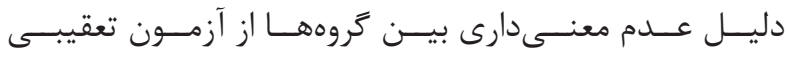
اسـتفاده نشــد (جــدول ع (؟).

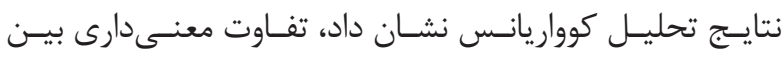

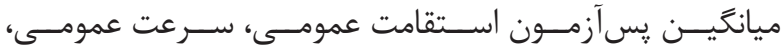

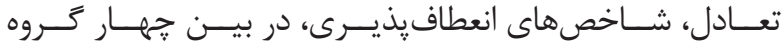

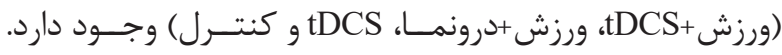

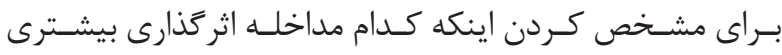

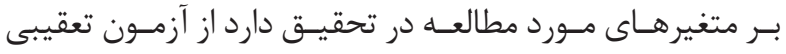

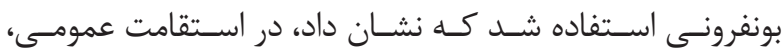

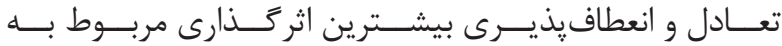

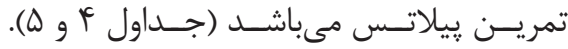

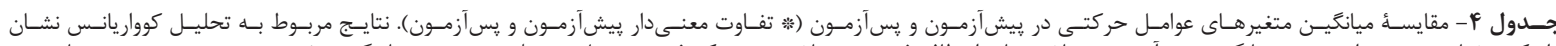

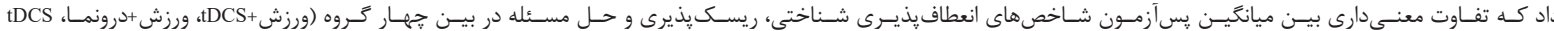

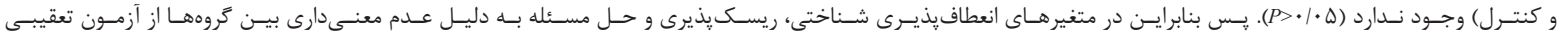

\begin{tabular}{|c|c|c|c|c|c|c|c|c|c|}
\hline \multicolumn{2}{|c|}{ مروه كثترل (ه نفر) } & \multicolumn{2}{|c|}{ (9) tDCS } & \multicolumn{2}{|c|}{ ورزش + دارونما (و نفر) } & \multicolumn{2}{|c|}{ ورزش+1DCS (نفر) } & \multirow[t]{2}{*}{ "مروه } & \multirow{5}{*}{ حركتى } \\
\hline بس آزمون & ييش آزمون & بس آزمون & ييش آزمون & بس آزمون & ييش آزمون & بس آزمون & ييش آزمون & & \\
\hline $11 \Delta / q \pm r r / \Lambda 1$ & $\|\Delta /\| \pm\|\| / Y \|$ & $\| \mathrm{V} / \mathrm{V} \pm \Delta \xi / \mathrm{r}$. & IIV/V & * $1 r \cdot / q \pm 11 / r v$ & $\|Q /\| \pm Y A / F V$ & $=119 / 11 \pm \wedge q / F q$ & $\|\xi /\| F \pm\|\| \cdot \Delta$ & عمومى استقامت & \\
\hline$r \cdot / \Delta \pm \Delta F / F A$ & $r \cdot|\Delta \pm \xi V / g|$ & $\mid V / \Delta \pm * \cdot / T F$ & $\mid G / \Delta \pm V A / F T$ & $r Y=|\Delta \pm *.| \Lambda \mid$ & $r \cdot / 9 \pm * * / F *$ & 다. $/ 9 \pm \Delta \& / 19$ & $1 \mathrm{~N} / \mathrm{V} \pm 9 \mathrm{~V} / \mathrm{H} G$ & أنعافي & \\
\hline$N / I \pm r / H$. & $N / 1 \pm r r / F 1$ & $\Lambda / r \pm * * / r$ & $\mathrm{~N} / \mathrm{r} \pm \mathrm{rr} / \cdot 9$ & $\omega q / / \pm \xi V / A V$ & $V / I \pm \Delta \Delta / V F$ & $* 9 / / \pm g \mathrm{~V} / \Delta$. & $\mathrm{N} / * \pm \mathrm{rV} / 99$ & تعادل & \\
\hline$F / \cdot \pm Y F / Y F$ & $F / \cdot \pm r \mid / r \Delta$ & $F / \cdot \pm r \Delta / Y F$ & $F / \bullet \pm Y V / Y G$ & $F / \bullet \pm \Psi|/ r|$ & $F / * \pm r g / r Y$ & $F / \cdot \pm 1 \cdot / \pi \Delta$ & $F / \cdot \pm \mid F / r V$ & سمرعى & \\
\hline
\end{tabular}

جدول ه- سطح معنى دارى متغيرهاى مورد اندازميرى در مراحل پِيش آزمون و پِآزمون عوامل حركتى.

\begin{tabular}{|c|c|c|c|}
\hline سطع معثى دارى & & & متثير \\
\hline$*+1+V$ & ورزش tDCS & \multirow{4}{*}{ استقامث عمومى } & \multirow{16}{*}{ عوامل حركتى } \\
\hline $20+1+0$ & ورزئ +دارونما & & \\
\hline+1199 & $\mathrm{tDCS}$ & & \\
\hline+1990 & كتبرل & & \\
\hline$*+1+r$ & ورزش+tDCS & \multirow{4}{*}{ انعطافيذيرى } & \\
\hline$* 2+1+1$ & ورزث+دارونما & & \\
\hline */TFV & tDCs & & \\
\hline +/VNT & كتبرل & & \\
\hline 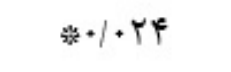 & ورزش tDCS & \multirow{4}{*}{ تعادل } & \\
\hline $3 *+1+1$ & ورزش+دارونما & & \\
\hline$+\mid F(\mid)$ & tDCS & & \\
\hline$+/ \Delta 9 F$ & كتبرل & & \\
\hline (r) & ورزش tDCS+ & \multirow{4}{*}{ سرعت عمومى } & \\
\hline + ITS & ورزث+دارونما & & \\
\hline H/T/G & tDCS & & \\
\hline + & كتبرل & & \\
\hline
\end{tabular}




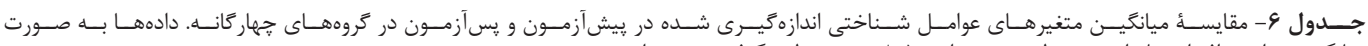

\begin{tabular}{|c|c|c|c|c|c|c|c|c|c|c|}
\hline \multicolumn{2}{|c|}{ كروهة كنترل (9 فقر) } & \multicolumn{2}{|c|}{ ( ) أنفر) IDCS } & \multicolumn{2}{|c|}{ 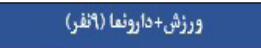 } & \multicolumn{3}{|c|}{ ورزش+DCS (iأفر) } & \\
\hline بس آزنون & بيش آنعون & بس آزنون & بيش آزمون & بس آزنون & بيش آزمون & بسل آزهون & \multicolumn{2}{|c|}{ بيش آزمون } & & \\
\hline$\cdot| \pm \Delta Y| Y \mid$ & $r / I \pm Y r / Y Y$ & $\cdot+\Delta \Delta / 11$ & $r / \cdot \pm r Y / A \gamma$ & $\cdot+ \pm \Delta|/|$ & $r / I \pm \cdot \cdot / / r$ & $\cdot / \pm \pm N \cdot 9$ & $Y / Y \pm \lambda / / F Y$ & تع تعاد مرالمل & \multirow{4}{*}{ شنعانتخى } & \multirow{9}{*}{ عُشانتى عوامل } \\
\hline $\mathrm{V} / \pm \pm \mathrm{V} / \cdot \mathrm{r}$ & $\cdot f \pm \mp N \cdot Y$ & $V \cdot \pm V F \mid \cdot r$ & $\Delta Y / \pm \pm \Delta \Delta q Y$ & $V \cdot \pm V Y \mid+Y$ & $F N F \pm 1 / / 11$ & $1 / + \pm V \mid+F$ & FNYFEIU/AF & باسخهاى & & \\
\hline $1 / \cdot \pm Y Q \cdot 9$ & $1 N Y \pm / 99 / 19$ & $\cdot+ \pm q \Delta / 1$. & $1+1 \pm \pm f \mid q \gamma$ & $V / \pm+\varphi r / 1$. & $10 / f \pm / 9 / 11$ & $1 / \cdot \pm+r / 11$ & 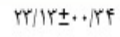 & خطامائ كلى & & \\
\hline$\cdot+ \pm r Y / \Delta$ & $\cdot / \Delta$. & $\cdot \mid \pm 11 / / Y Y$ & $\cdot \mid \pm f f F / d r$ & $\cdot+ \pm r r / \Delta \cdot$ & $1 / 1 \pm Y Y / Y$. & $1 / + \pm r \Delta \cdot 4$ & $V / \pm \pm N F A$ & خام خطاى & & \\
\hline$+\mid + \pm v g /+r$ & $\Delta / \pm F r / M$ & $\cdot| \pm \pm Y Y|+Y$ & $91 . \pm Y Y / \Delta 9$ & $+\cdot \pm \mathrm{VQ} /+\mathrm{Y}$ & $\Delta / \cdot \pm 11 / \Delta f$ & $\cdot \mid+ \pm \varepsilon q / \cdot r$ & $V \mid \pm \Psi Y N A G$ & 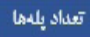 & \multirow{3}{*}{ حل مستلك } & \\
\hline$f \mid \cdot \pm G Y / Y Y$ & $F / + \pm r Y / Y Y$ & $Y| + \pm 9 F| Y \mid$ & $F /+$ YNYSB & $F \mid + \pm F Y / Y Y$ & $f|\cdot \pm \cdot Y| q f$ & $Y / + \pm V q / Y F$ & $f|+ \pm| d \mid r y$ & Lollat & & \\
\hline fMr/ffetr/lo & $f \Delta F / Y 1+ \pm 9 \Delta+y$ & $f g \pm r f f \mid \cdot v$ & $Y F \Delta Y+ \pm 9 N 91$ & $K Y 1 / F F \pm N F F$ & $F \cdot q / Y Y K$ NQ $+\mid \xi Y Y$ & $F \wedge \pm 1 f \cdot V Y$ & $Y+\Delta \pm F Y Y] \Lambda \Delta$ & زمان زمان (8) & & \\
\hline$\cdot \cdot \pm N / / Y$ & $9 / 1 \pm 91 / / 8$ & $\cdot \cdot \pm 9 V / 1 Y$ & $1 / \Delta \pm .9 / 9 \mathrm{~V}$ & $\cdot \cdot \pm \Lambda Y / 1 \Lambda$ & $F|Y \pm V| r|9|$ & $\cdot / + \pm 9 F / 1 Y$ & $11 / q \pm \Delta r / \gamma f$ & AV & ريسكباقيوي & \\
\hline$\cdot \mid \pm \pm M^{r} / 11$ & $Y / Y \pm Y G / Y \Delta$ & $\cdot \mid+ \pm\langle F| \cdot 9$ & Ff. $\pm F Y / F Y$ &.$/ . \pm V Y / 10$ & $r \mid + \pm V / a r$ & $\cdot| \pm \pm \theta /|$. & $y / f \pm \lambda y / f f$ & $\mathrm{UA}$ & (آزنون بارث) & \\
\hline
\end{tabular}

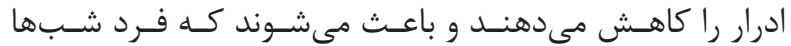

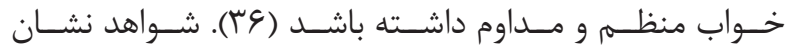

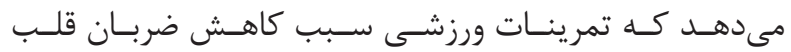

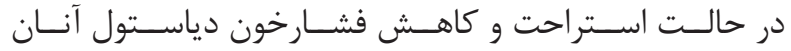

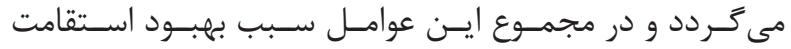

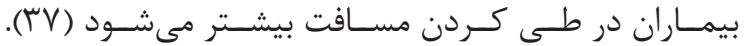

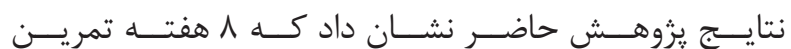

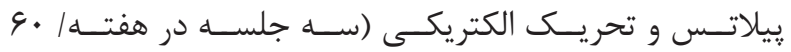

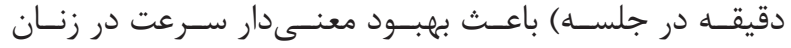

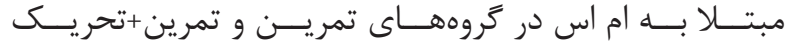

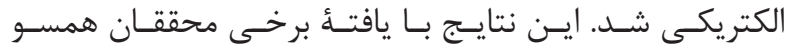

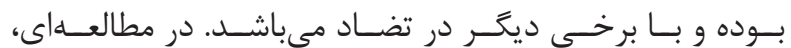

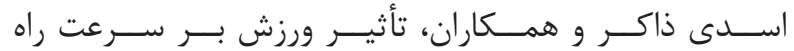

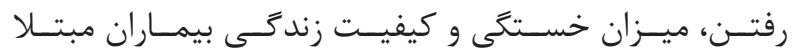

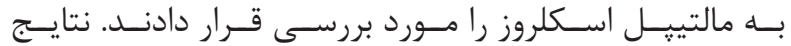

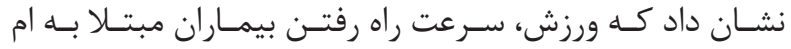

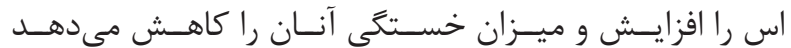

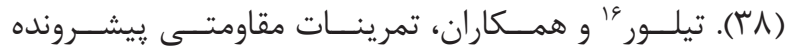

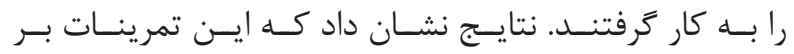

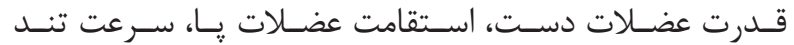

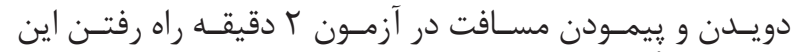

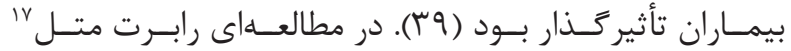

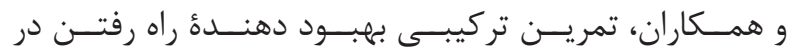

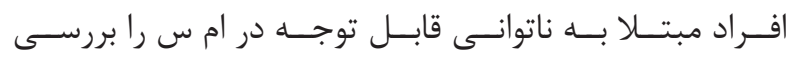

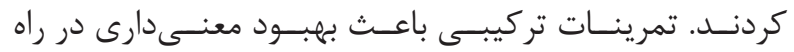

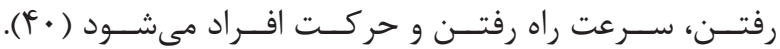

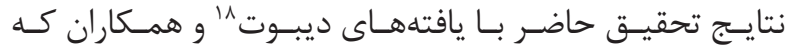

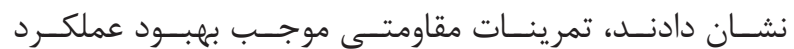

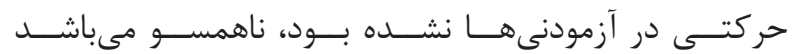

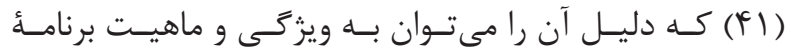

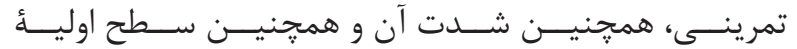

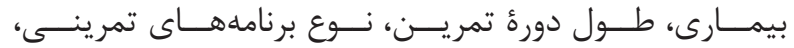

${ }^{14}$ Niuman

${ }^{15}$ Detmers

${ }^{16}$ Taylor
بحث و نتيجه گيرى

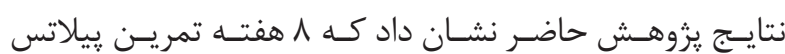

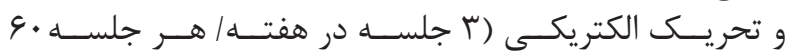

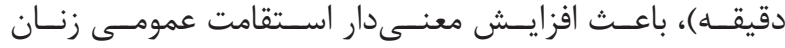

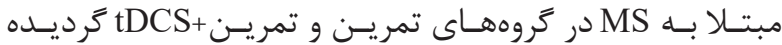

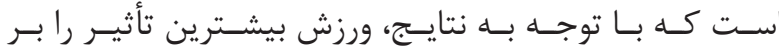

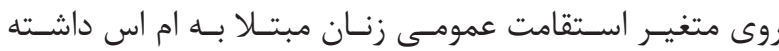

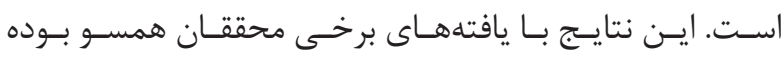

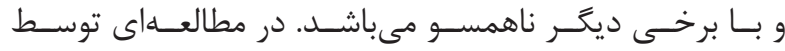

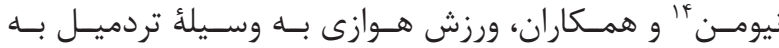

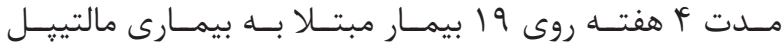

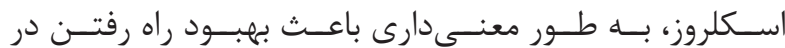

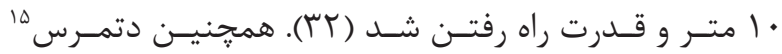

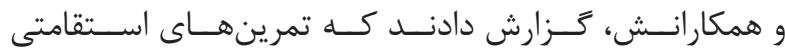

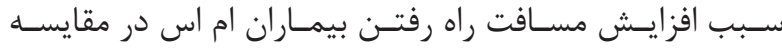

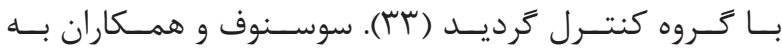

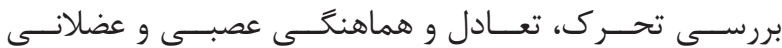

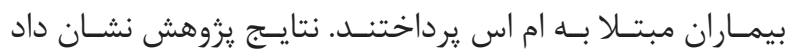

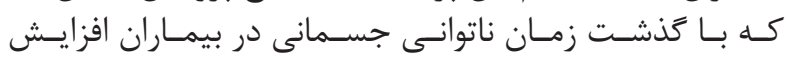

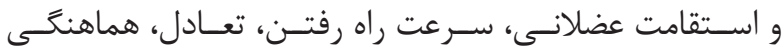

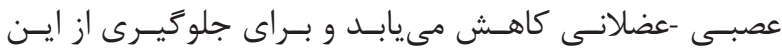

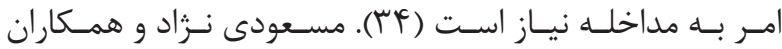

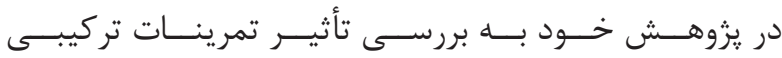

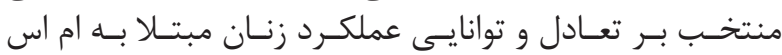

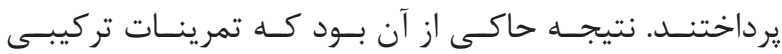

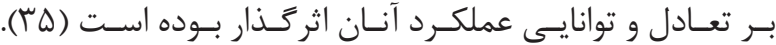

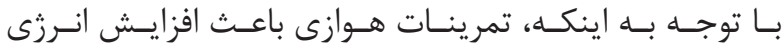

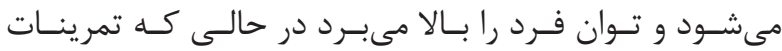

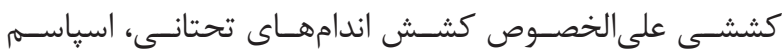

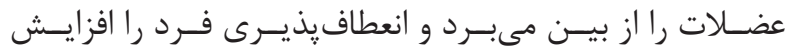

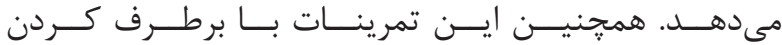

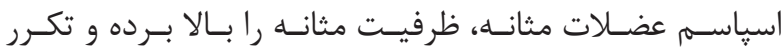

${ }^{17}$ Motl

${ }^{18}$ Debut 


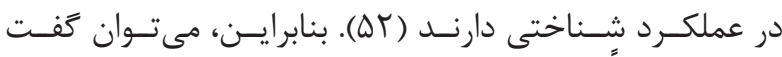

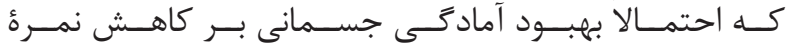

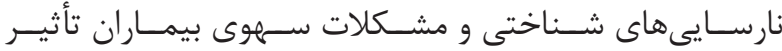

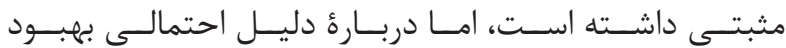

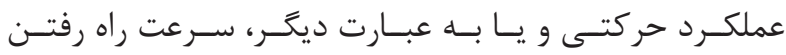

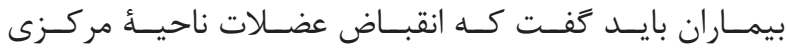

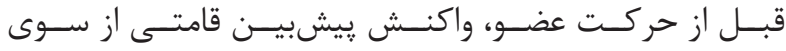

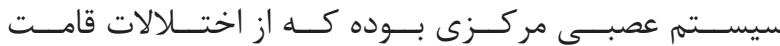

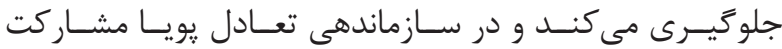

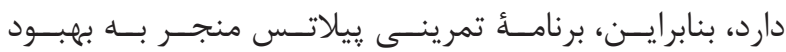

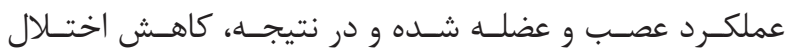

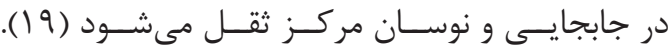

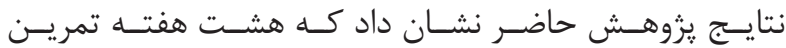

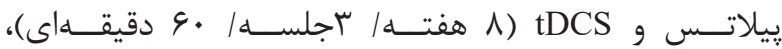

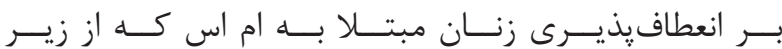

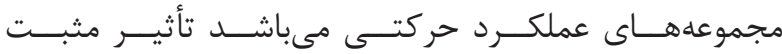

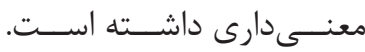

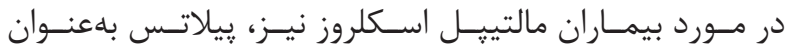

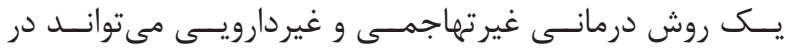

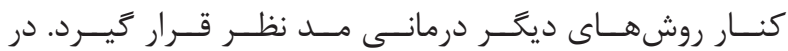

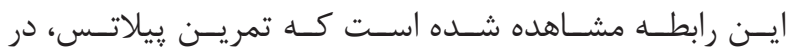

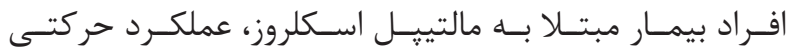

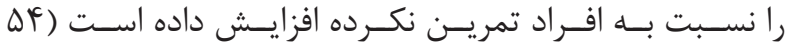

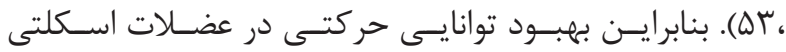

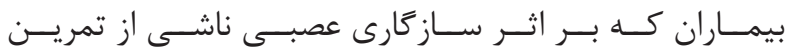

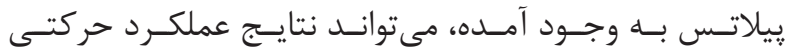

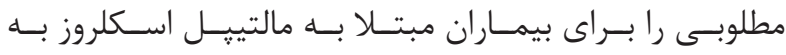

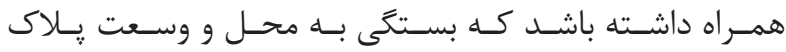

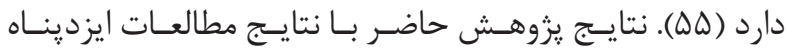

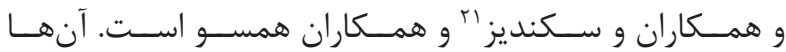

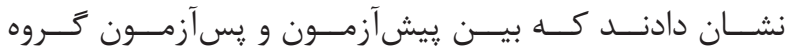

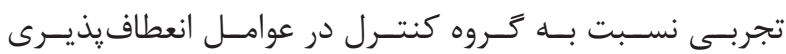

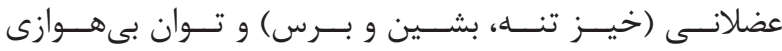

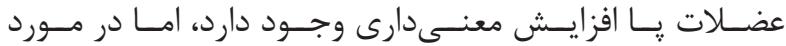

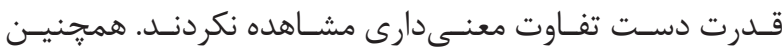

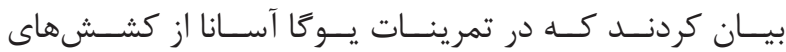

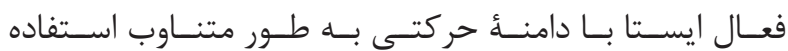

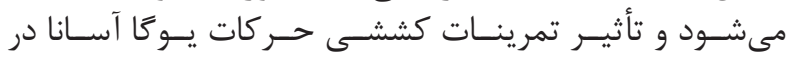

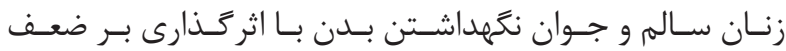

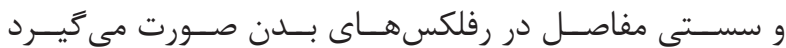

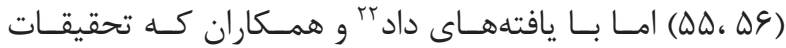

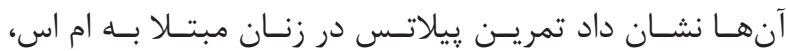

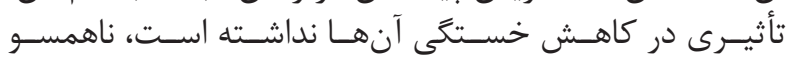

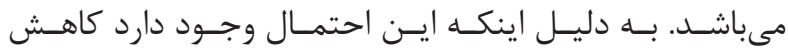

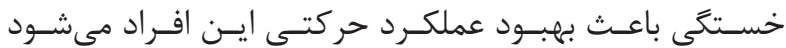
(DV، DA)

${ }^{19}$ Lebietaz

${ }^{20}$ Freeman

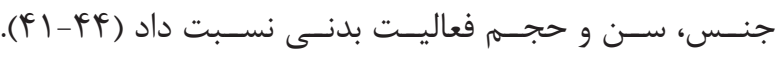

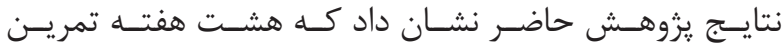

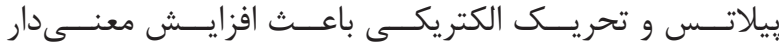

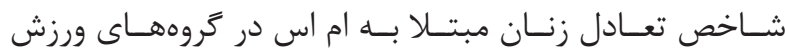

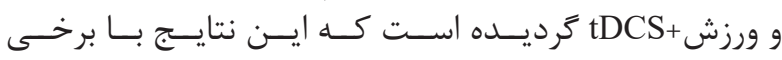

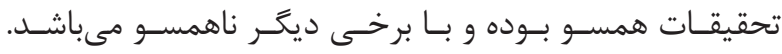

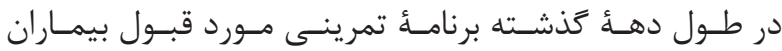

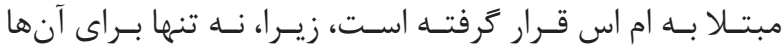

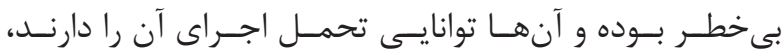

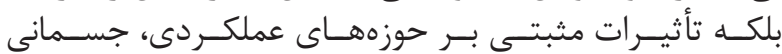

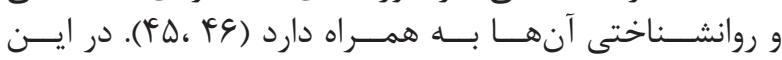

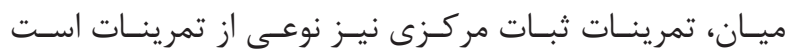

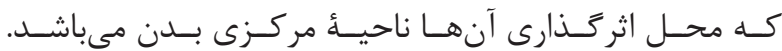

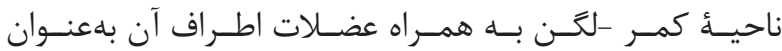

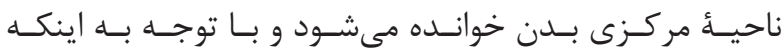

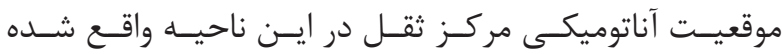

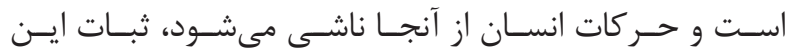

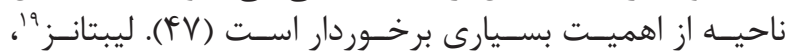

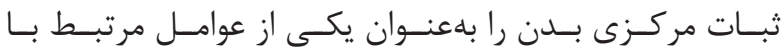

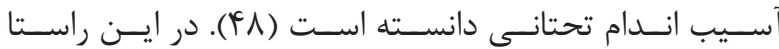

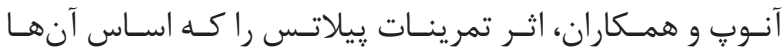

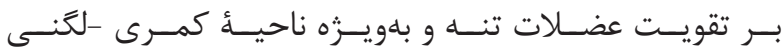

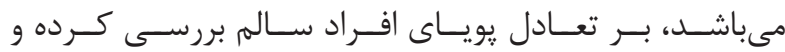

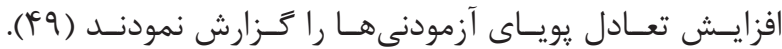

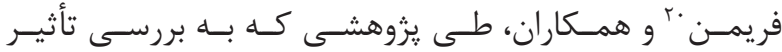

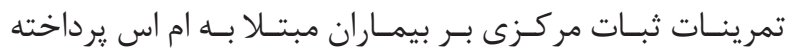

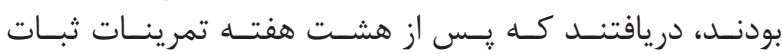

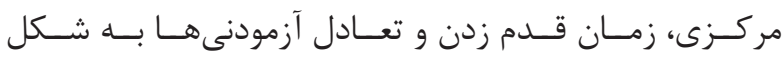

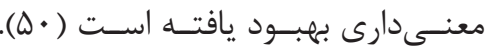

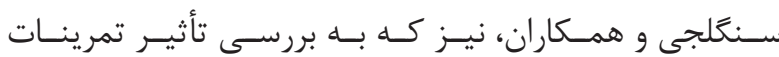

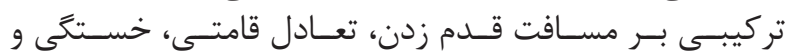

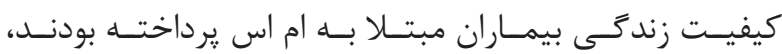

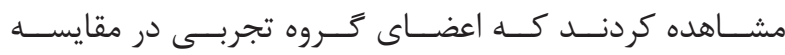

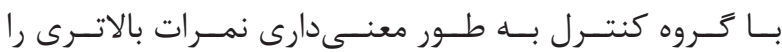

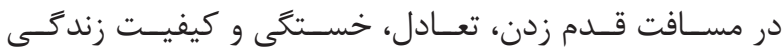

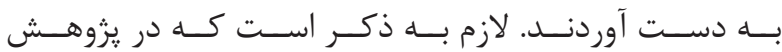

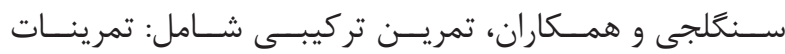

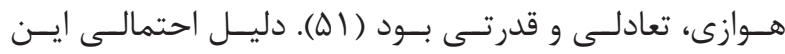

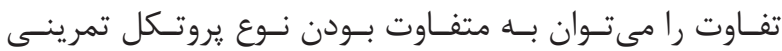

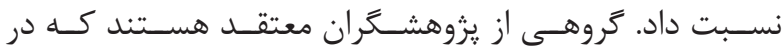

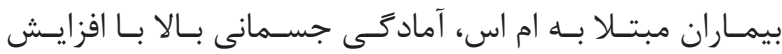

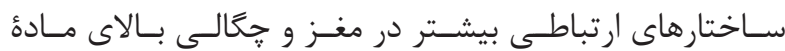

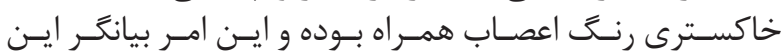

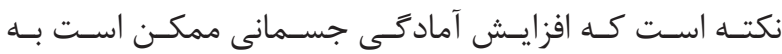

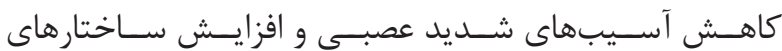

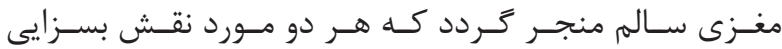

${ }^{21}$ Sekendiz

${ }^{22}$ Dodd 


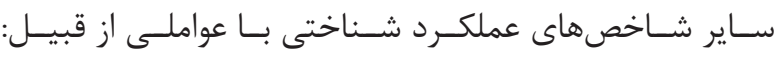

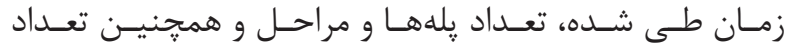

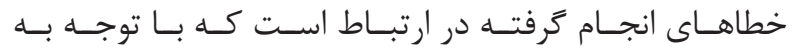

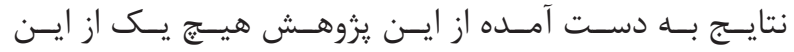

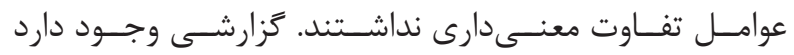

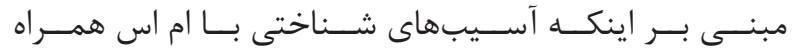

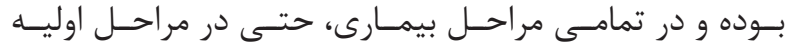

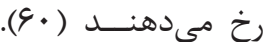

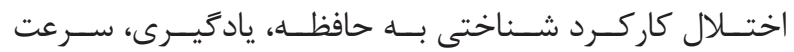

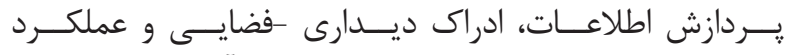

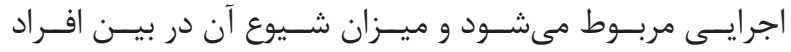

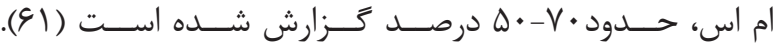

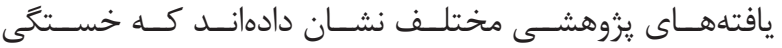

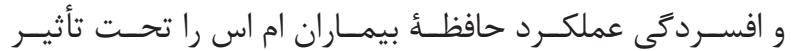

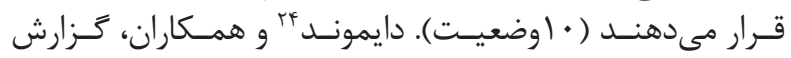

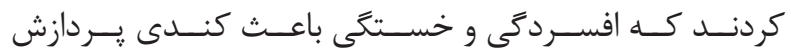

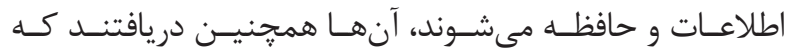

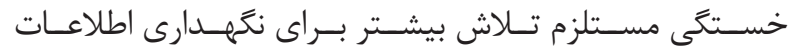

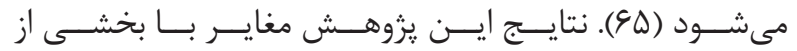

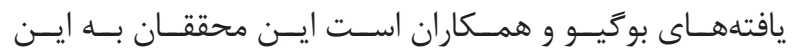

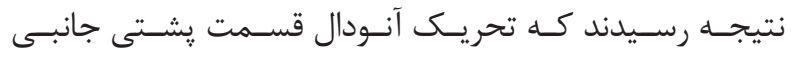

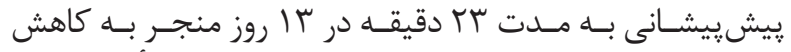

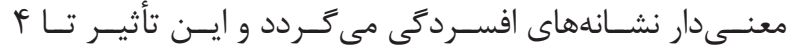

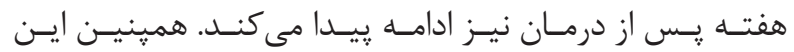

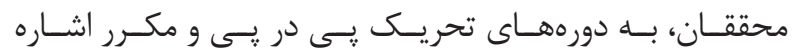

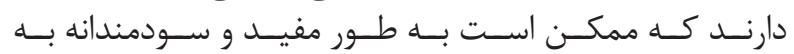

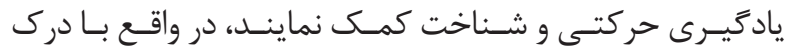

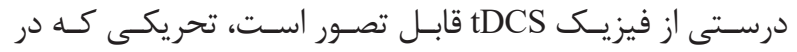

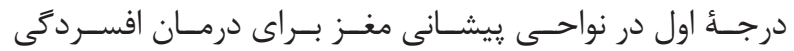

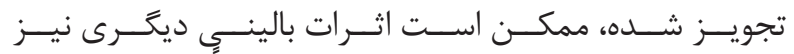

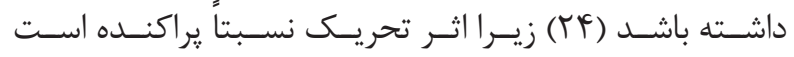

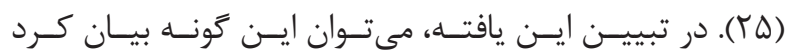

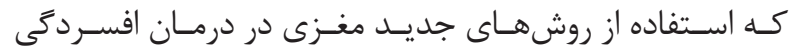

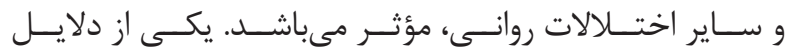

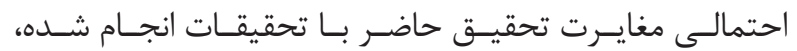

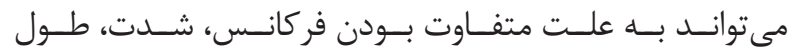

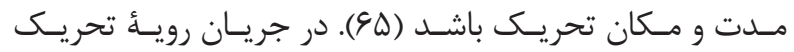

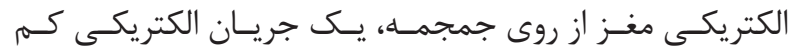

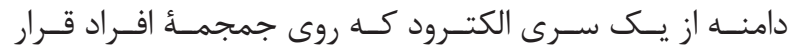

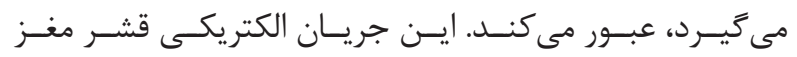

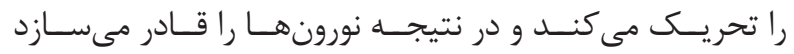

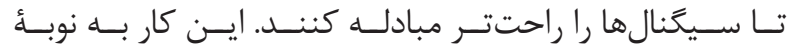

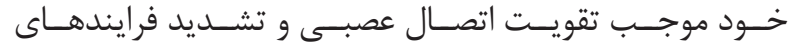

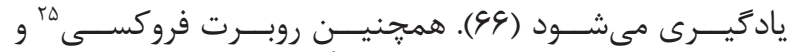

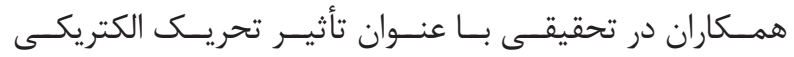

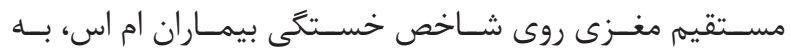

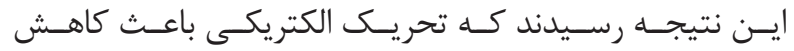

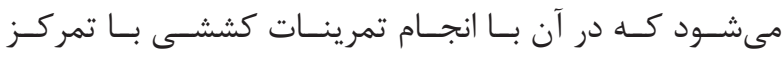

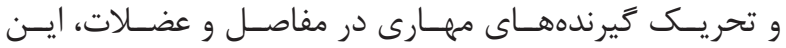

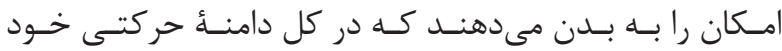

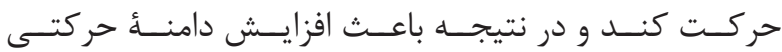

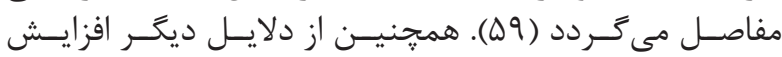

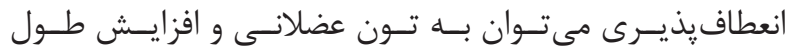

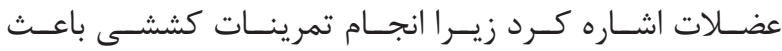

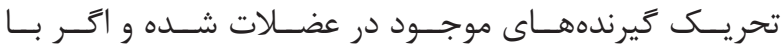

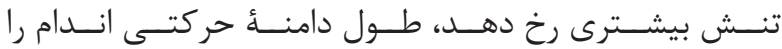

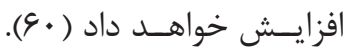

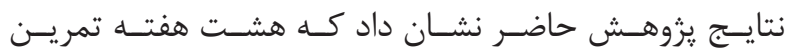

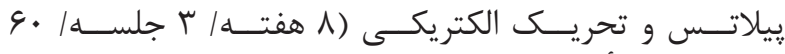

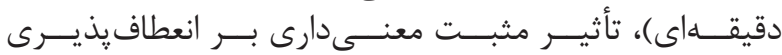

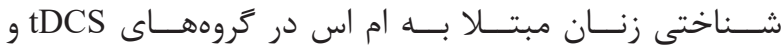

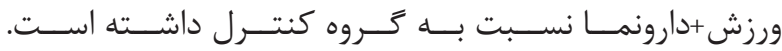

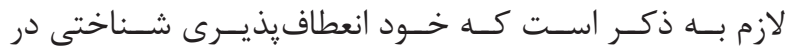

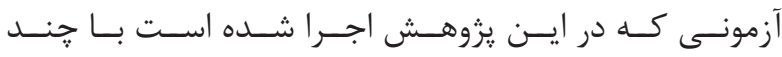

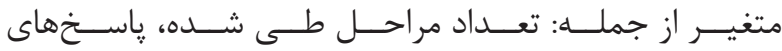

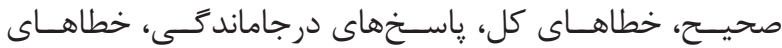

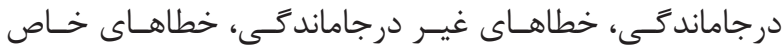

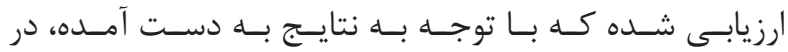

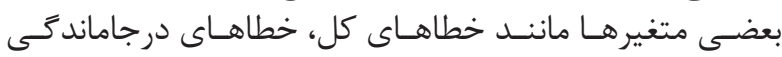

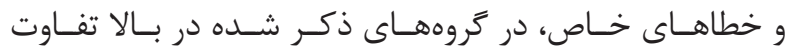

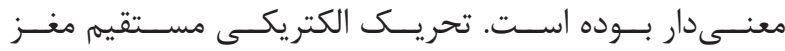

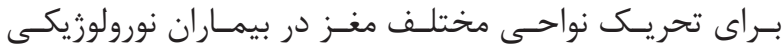

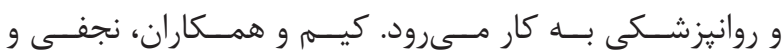

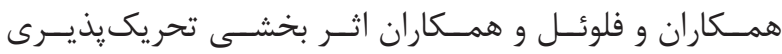

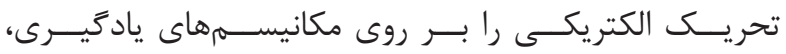

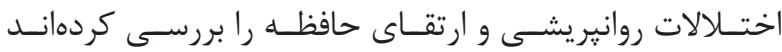

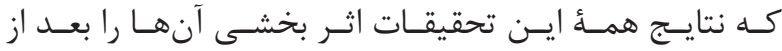

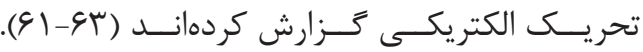

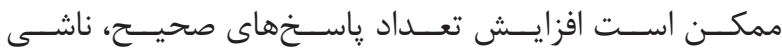

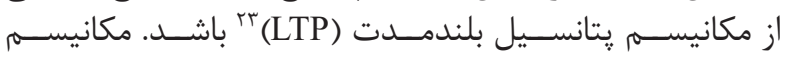

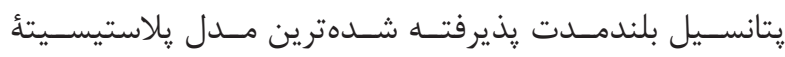

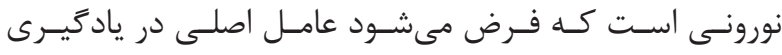

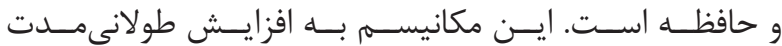

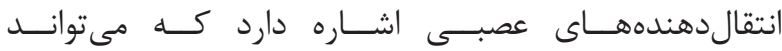

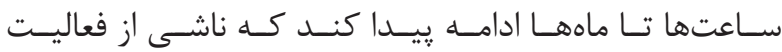

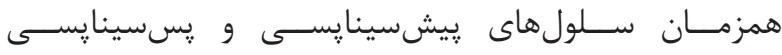

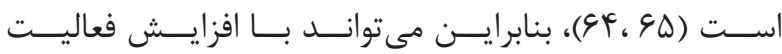

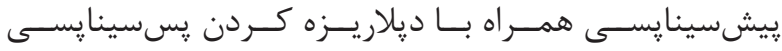

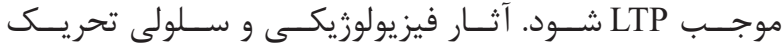

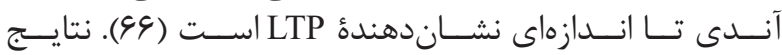

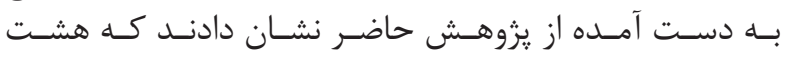

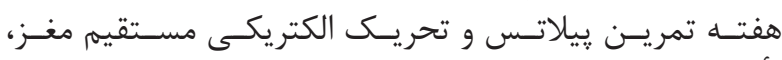

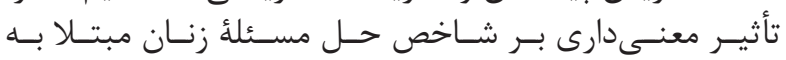

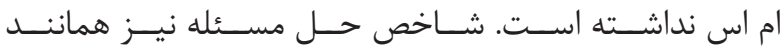

${ }^{23}$ Long term potentioal

${ }^{24}$ Diamond

${ }^{25}$ Roberta Ferrucci 


$$
\begin{aligned}
& \text { درمانـى غيرتهاجمـى، عامـل مؤثـــى بــر بهبــود توانايسى انجــام }
\end{aligned}
$$

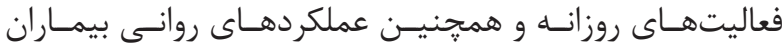

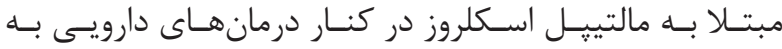

$$
\begin{aligned}
& \text { ايسن افـراد توصيـهـ شـوند. } \\
& \text { تشكر و قدردانى }
\end{aligned}
$$

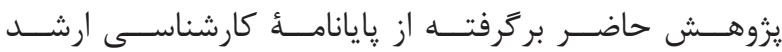

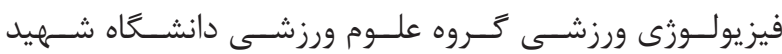

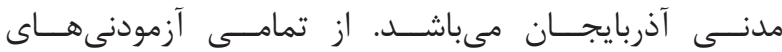

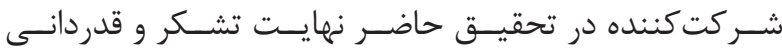

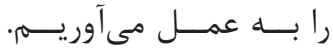

1. Ashtari F, Shaygannejad V, Heidari F, Akbari M. Prevalence of familial multiple sclerosis in Isfahan, Iran. Journal of Isfahan Medical School. 2011; 29(138): 555-61.

2. Soltani M, Hejazi SM, Nouriyan A, Zendel A, Ashkani M. Effects of aerobic training on improving the water balance in selected patients with multiple sclerosis. Journal of Nursing and Midwifery, Mashhad. 2009; 9(2): 107-13.

3. Farma BS. Introduction to multiple sclerosis 1, translation company bayer pharma shrink office. Tehran: Jalal Publication. 2010.

4. Costello K, Kennedy P, Scanzillo J. Recognizing nonadherence in patients with multiple sclerosis and maintaining treatment adherence in the long term. Medscape J Med. 2005; 10(9): 225.

5. Shanazari Z, Marandi SM, Minasian V. Effect of 12-week pilates and aquatic training on fatigue in women with multiple sclerosis. Journal of Mazandaran University of Medical Sciences. 2013; 23(98): 257-64.

6. Schwartz CE, Laitin E, Brotman S, LaRocca N. Utilization of unconventional treatments by persons with MS: Is it alternative or complementary? Neurology. 1999; 52(3): 626-9.

7. Mohr DC, Hart SL, Julian L, Cox D, Pelletier D. Association between stressful life events and exacerbation in multiple sclerosis: a meta-analysis. Bmj. 2004; 328(7442): 731 .

8. Golan D, Somer E, Dishon S, Cuzin-Disegni L, Miller A. Impact of exposure to war stress on exacerbations of multiple sclerosis. Ann Neurol. 2008; 64(2): 143-8.

9. Brown LA, Kerr CJ, Whiting P, Finer N, McEneny $\mathrm{J}$, Ashton T. Oxidant stress in healthy normal-weight, overweight, and obese individuals. Obesity. 2009; 17(3): 160-6.

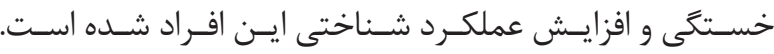

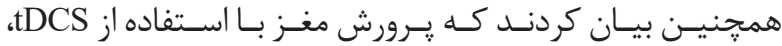

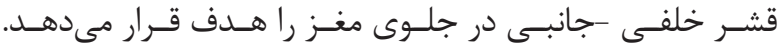

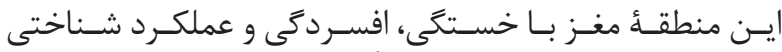

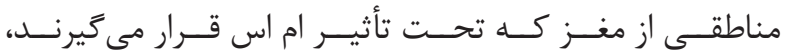

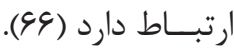

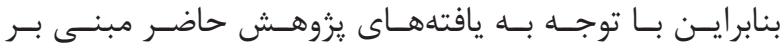

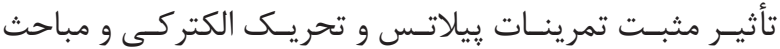

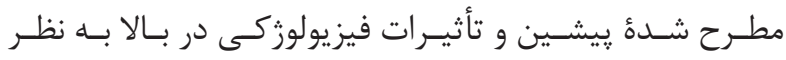

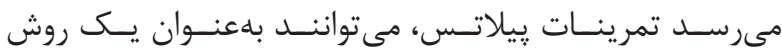

$$
\begin{aligned}
& \text { منابع }
\end{aligned}
$$

10. Willoughby KL, Dodd KJ, Shields N. A systematic review of the effectiveness of treadmill training for children with cerebral palsy. Disabil Rehabil. 2009; 31(24): 1971-9.

11. Fresco DM, Rytwinski NK, Craighead LW. Explanatory flexibility and negative life events interact to predict depression symptoms. Journal of Social and Clinical Psychology. 2007; 26(5): 595-608.

12. Cameron JI, Shin JL, Williams D, Stewart DE. A brief problem-solving intervention for family caregivers to individuals with advanced cancer. J Psychosom Res. 2004; 57(2): 137-43.

13. Mohr DC, Pelletier D. A temporal framework for understanding the effects of stressful life events on inflammation in patients with multiple sclerosis. Brain Behav Immun. 2006; 20(1): 27-36.

14. Mitsonis CI, Zervas IM, Mitropoulos PA, Dimopoulos NP, Soldatos CR, Potagas CM, et al. The impact of stressful life events on risk of relapse in women with multiple sclerosis: a prospective study. Eur Psychiatry. 2008; 23(7): 497-504.

15. Buljevac D, Hop W, Reedeker W, Janssens A, Van Der Meché F, Van Doorn P, et al. Self reported stressful life events and exacerbations in multiple sclerosis: prospective study. Bmj. 2003; 327(7416): 646.

16. Muscolino JE, Cipriani S. Pilates and the"powerhouse". Journal of Bodywork and Movement Therapies. 2004; 8(1): 15-24.

17. Heese O, Schmidt M, Nickel S, Berger H, Goldbrunner $\mathrm{R}$, Tonn J, et al. Complementary therapy use in patients with glioma: an observational study. Neurology. 2010; 75(24): 2229-35.

18. Durstine JL, Moore GE, Patricia L, Roberts SO. ACSM's exercise management for persons with chronic diseases and disabilities. $3^{\text {th }}$ ed. American College of 


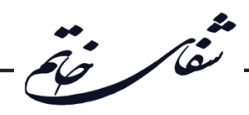

Sports Medicine. 2008.

19. Alizamani S, Ghasemi Gh, Karimi A, Salehi H. Pilates exercises on pain and general health of female patients with chronic low back pain. Rehabilitation Sciences Research. 2011; 7(1): 117.

20. Romberg A, Virtanen A, Ruutiainen T, Seppanen, A. Effects of a 6-month exersice program on patients with Multiple sclerosis. Neurology. 2004; 63(11): 2034-8.

21. Mostert S, Kesselring J. Effects of a short -term exercise training program on aerobic fitness, health perception and level of subject with multiple sclerosis. Mult Scler. 2002; 8(2): 161-8.

22. Rampello A, Franceschini M, Piepoli M, Antenucci R, Lenti G, Olivieri D, et al. Effect of aerobic training on walking capacity and maximal exercise tolerance in patients with multiple sclerosis: A randomized crossover controlled study. Phys Ther. 2007; 85(7): 545-55.

23. Clark VP, Coffman BA, Trumbo MC, Gasparovic C. Transcranial direct current stimulation (tDCS) produces localized and specific alterations in neurochemistry: a $1 \mathrm{H}$ magnetic resonance spectroscopy study. Neurosci Lett. 2011; 500(1): 67-71.

24. Boggio PS, Bermpohl F, Vergara AO, Muniz AL, Nahas FH, Leme PB, et al. Go-no-go task performance improvement after anodal transcranial DC stimulation of the left dorsolateral prefrontal cortex in major depression. J Affect Disord. 2007; 101(1-3): 91-8.

25. Andrews SC, Hoy KE, Enticott PG, Daskalakis ZJ, Fitzgerald PB. Improving working memory: the effect of combining cognitive activity and anodal transcranial direct current stimulation to the left dorsolateral prefrontal cortex. Brain Stimul. 2011; 4(2): 84-9.

26. Dröge W. Aging-related changes in the thiol/ disulfide redox state: implications for the use of thiol antioxidants. Exp Gerontol. 2002; 37(12): 1333-45.

27. Anderson BJ, Rapp DN, Baek DH, McCloskey DP, Coburn-Litvak PS, Robinson JK. Exercise influences spatial learning in the radial arm maze. Physiol Behav. 2000; 70(5): 425-9.

28. Mohamadi DZ, Nezakatolhossaini M, Esfarjani F, Etemadifar M. The effect of 8-week Pilates training on motor function and depression in subjects with Multiple Sclerosis (MS). Journal of Research in Rehabilitation Sciences. 2013; 9(2): 308-17.

29. Dalgas U, Stenager E, Ingemann-Hansen T. Multiple sclerosis and physical exercise: recommendations for the application of resistance-, endurance-and combined training. Mult Scler. 2008; 14(1): 35-53.

30. da Silva MC, Conti CL, Klauss J, Alves LG, do Nascimento Cavalcante HM, Fregni F, et al. Behavioral effects of transcranial direct current stimulation (tDCS) induced dorsolateral prefrontal cortex plasticity in alcohol dependence. J Physiol Paris. 2013; 107(6): 493502 .

31. Zalewski K. Exploring barriers to remaining physically active: a case report of a person with multiple sclerosis. J Neurol Phys Ther. 2007; 31(1): 40-5.

32. Newman M, Dawes H, Van den Berg M, Wade D, Burridge J, Izadi H. Can aerobic treadmill training reduce the effort of walking and fatigue in people with multiple sclerosis: a pilot study. Mult Scler. 2007; 13(1): 113-9.

33. Dettmers C, Sluzmann M, Ruchay-Ploss I, Gluter R, Vieten M. Endurance exercise important walking distance in MS patient with fatigue. Acta Neurol Scand. 2009; 120(4): 251-7.

34. Sosnoff JJ, Socie MJ, Boes MK, Sandroff BM, Pula $\mathrm{JH}$, Suh Y, et al. Mobility, balance and falls in persons with multiple sclerosis. PLoS One. 2011; 6(11): 1-5.

35. Masuodi Nejad M, Shivani H, Hosseini F. Effects of selected combined training on balance and functional capacity in women with multiple sclerosis. World Applied Sciences Journal. 2012; 16(7): 1019-26.

36. Müller G. Take-over: multiple mechanisms of interadipocyte communication. Oxford University Press; 2011.

37. Patanella AK, Zinno M, Quaranta D, Nociti V, Frisullo G, Gainotti G, et al. Correlations between peripheral blood mononuclear cell production of BDNF, TNF-alpha, IL-6, IL-10 and cognitive performances in multiple sclerosis patients. J Neurosci Res. 2010; 88(5): 1106-12.

38. Asadizaker M, Majdinasab N, Atapour M, Latifi S, Babadi M. Effect of exercise on walking speed, fatigue and quality of life in patients with multiple sclerosis. Jundishapur Scientific Medical Journal. 2010; 9(2): 189-98.

39. Taylor N, Dodd K, Prasad D, Denisenko S. Progressive resistance exercise for people with multiple sclerosis. Disabil Rehabil. 2006; 28(18): 1119-26.

40. Motl RW, Smith DC, Elliott J, Weikert M, Dlugonski D, Sosnoff JJ. Combined training improves walking mobility in persons with significant disability from 
multiple sclerosis: a pilot study. J Neurol Phys Ther. 2012; 36(1): 32-7.

41. DeBolt LS, McCubbin JA. The effects of home-based resistance exercise on balance, power, and mobility in adults with multiple sclerosis. Arch Phys Med Rehabil. 2004; 85(2): 290-7.

42. McCaskey A. The effects of core stability training on star excursion balance test and global core muscular endurance. University of Toledo. 2011.

43. Czaprowski D, Afeltowicz A, Gębicka A, Pawłowska P, Kędra A, Barrios C, et al. Abdominal muscle EMGactivity during bridge exercises on stable and unstable surfaces. Phys Ther Sport. 2014; 15(3): 162-8.

44. Laitiff AA, Teoh SL, Das S. Wound healing in diabetes mellitus: traditional treatment modalities. Clin Ter. 2010; 161(4): 359-64.

45. Ahmadi R, Hasan D, Hosin BA. The effect of 6 weeks core stabilization training program on the balance in mentally retarded students. International Journal of Sport Studies. 2012; 2(10): 496-501.

46. Cradock KA, ÓLaighin G, Finucane FM, Gainforth HL, Quinlan LR, Ginis KAM. Behaviour change techniques targeting both diet and physical activity in type 2 diabetes: A systematic review and meta-analysis. Int J Behav Nutr Phys Act. 2017; 14(1): 18. doi: 10.1186/ s12966-016-0436-0.

47. Aminoff MJ. Aminoff's electrodiagnosis in clinical neurology. 6th ed. Expert Consult-Online and Print: Elsevier Health Sciences. 2012. p. 890.

48. Liebetanz D, Fregni F, Monte-Silva KK, Oliveira MB, Amâncio-dos-Santos A, Nitsche MA, et al. Aftereffects of transcranial direct current stimulation (tDCS) on cortical spreading depression. Neurosci Lett. 2006; 398(1-2): 85-90.

49. Anoop A, Kalpana Z, Jitender M, Kumar S. Effect of core stabilization training on dynamic balance in non-professional sports players. Indian Journal of Physiotherapy and Occupational Therapy-An International Journal. 2010; 4(4): 18-22.

50. Freeman J, Fox E, Gear M, Hough A. Pilates based core stability training in ambulant individuals with multiple sclerosis: protocol for a multi-centre randomised controlled trial. BMC Neurol. 2012; 12(1) 19. doi: 10.1186/1471-2377-12-19.

51. Sangalaji B, Salimi Y, Khatpour M, Mansouri T, Ashrafinia F, Esmailzadeh N, et al. The relationship between disability and quality of life in patients with multiple sclerosis MS Tehran. Journal of Health and Development. 2013; 2(3): 203.

52. Widener GL, Allen DD, Gibson-Horn C. Randomized clinical trial of balance-based torso weighting for improving upright mobility in people with multiple sclerosis. Neurorehabil Neural Repair. 2009; 23(8): 784-91.

53. Katsavos S, Anagnostouli M. Biomarkers in multiple sclerosis: an up-to-date overview. Mult Scler Int. 2013; 2013: doi: 10.1155/2013/340508.

54. Singh RS. After obama: renewing American leadership, restoring global order: Cambridge University Press; 2016.

55. Eizad Panah S, Marandi M, Bambaei-chi E, Nazarian $\mathrm{AB}$, Noorian KH. The effect of yoga training on some of physical fitness factors in non-athletes healthy women, Sport Physiology. Res Sport Sci. 2009; 23(6): 37-48.

56. Sekendiz B, Cug M, Korkusuz F. Effect of swiss-ball core strength training on strength, endurance, flexibility and balance in sedentary women. J Strength Cond Res. 2010; 24(11): 3032-40.

57. Dodd K, Taylor N, Denisenko S, Prasad D. A qualitative analysis of a progressive resistance exercise programme for people with multiple sclerosis. Disabil Rehabil. 2006; 28(18): 1127-34.

58. Cerasa A, Tongiorgi E, Fera F, Gioia MC, Valentino $\mathrm{P}$, Liguori $\mathrm{M}$, et al. The effects of BDNF Val66Met polymorphism on brain function in controls and patients with multiple sclerosis: an imaging genetic study. Behavioural Brain Research. 2010; 207(2): 377-86.

59. Chen W, Xu-Hong H, Zhang M-L, Yu-Qian B, YuHua Z, Zhong W-H, et al. Comparison of body mass index with body fat percentage in the evaluation of obesity in Chinese. Biomedical and Environmental Sciences. 2010; 23(3): 173-9.

60. Ebrahimi H, Blaouchi R, Eslami R, Shahrokhi M. Effect of 8-week core stabilization exercises on low back pain, abdominal and back muscle endurance in patients with chronic low back pain due to disc herniation. Physical Treatments-Specific Physical Therapy Journal. 2014; 4(1): 25-32.

61. Kim S, Stephenson MC, Morris PG, Jackson SR. tDCS-induced alterations in GABA concentration within primary motor cortex predict motor learning and motor memory: a $7 \mathrm{~T}$ magnetic resonance spectroscopy study. Neuroimage.2014; 99: 237-43. 
62. Najafi Dolatabad Sh, Noureyan kh, Najafi Dolatabad A, Mohebbi Z. The effect of Yoga techniques on quality of life among women with Multiple Sclerosis. Hormozgan University of Medical Sciences. 2010; 16(3): 142-50.

63. Floel A. tDCS-enhanced motor and cognitive function in neurological diseases. Neuroimage. 2014; 85(3): 934-47.

64. Kuo MF, Paulus W, Nitsche MA. Therapeutic effects of non-invasive brain stimulation with direct currents
(tDCS) in neuropsychiatric disease. NeuroImage. 2014; 85(3): 948-60.

65. Diamon BJ, Johnson SK, Kaufman M, Graves L. Relationships between infor. mation processing, depression, fatigue and cognition in multiple sclerosis. Arch Clin Neuropsychol. 2008; 23(2): 189-99.

66. Ferrucci R, Vergari M, Cogiamanian F, Bocci T, et al. Transcranial direct current stimulation (tDCS) for fatigue in multiple sclerosis. NeuroRehabilitation. 2014; 34: $121-7$. 